\title{
The Untargeted Capability of NMR Recognizes Adulterated Natural Products
}

Seon Beom Kim, Jonathan Bisson, J. Brent Friesen, Luca Bucchini, Stefan Gafner, David C. Lankin, Shao-Nong Chen, Guido F. Pauli, James B. McAlpine* 


\section{ABSTRACT}

Curcuma longa (turmeric) has a long ethnomedical background for common ailments, and Dietary Supplements (DS) labelled as "Curcumin" (CDS) are a highly visible portion of today's selfmedication market. Due to cost pressure, these CDS products are affected by economically motivated adulteration with synthetic curcumin and are associated with unexpected toxicological issues due to "residual" impurities. Using a combination of targeted and untargeted (phyto)chemical analysis, this study investigated the botanical integrity of two commercial "turmeric" CDS with vitamin and other additives that were associated with reported clinical cases of hepatotoxicity. Analyzing multi-solvent extracts of the CDS by $100 \%$ quantitative ${ }^{1} \mathrm{H}$ NMR (qHNMR), alone and in combination with countercurrent separation (CCS), provided chemical fingerprints that allowed both the targeted identification and quantification of declared components and the untargeted recognition of adulteration. While confirming the presence of curcumin as a major constituent, the universal detection capability of NMR identified significant residual impurities. While the loss free nature of CCS captured a wide polarity range of declared and unwanted chemical components and increased dynamic range, (q)HNMR determined their mass proportions and chemical constitutions. The results demonstrate that NMR can recognize undeclared constituents even if they represent a relatively minor gap in the mass balance of a DS product. The chemical information associated with the missing $4.8 \%$ and $7.4 \%(\mathrm{~m} / \mathrm{m})$ in the two commercial samples, exhibiting an otherwise adequate curcumin content of $95.2 \%$ and $92.6 \%$, pointed to a product integrity issue and adulteration with undeclared synthetic curcumin. Impurities from synthesis are most plausibly the cause of the observed adverse clinical effects. The study exemplifies how the simultaneously targeted and untargeted analytical principle of $100 \%$ qHNMR method, performed with entry-level instrumentation (400 MHz), can enhance the safety of DS by identifying adulterated, non-natural "natural" products. 


\section{INTRODUCTION}

The global marketplace for dietary supplements (DS) is extensive at an estimated $\$ 8+\mathrm{B}$ in U.S. sales and $\$ 200+\mathrm{B}$ globally. It continues to grow and is one of the mainstays of commercial natural products (NPs) other than foods. It is well established that plant-derived DS such as crude plant materials, their extracts, and products derived from them are chemically complex. Their composition reflects the metabolome(s) of the producing organism(s), i.e., the source plant(s), their associated microorganisms, and the processing methods used. In contrast to this widely acknowledged complexity, reductionist approaches continue to prevail in both the chemical analysis and biological evaluation of DS. In fact, the majority of contemporary standardization protocols and (botanical) product integrity analyses, including compendial and regulatory methods, are limited to only one or very few readily accessible phytochemicals, often functioning as chemotaxonomic rather than pharmacologically relevant markers, and tend to neglect bioactive and metabolically variable components of the DS. This already concerning (phyto)chemical reductionism translates into a potentially even more problematic biological reductionism: the majority of biological studies with DS and major constituents of DS focus on a relatively small number of "prominent" compounds. This leads to overemphasis of the perceived therapeutic potential of relatively few natural molecules, which "the literature" describes as having a myriad of purported beneficial biological properties. However, more plausibly, the majority of these overly prominent molecules should be considered IMPs, i.e., Invalid/Improbable/Interfering Metabolic Panaceas. $^{1-3}$

The Importance of "Yellow" Terminology - Label vs. Content. The term "curcumin" has been utilized - often confusingly - to describe five different types of materials, all of which are being marketed as "curcumin"-containing dietary supplements $(\mathrm{CDS}):^{3}$ (i) turmeric $(\mathrm{T})$, the raw turmeric (Curcuma longa) rhizome; (ii) turmeric extract (TE), the solvent extract of dried or fresh C. longa rhizomes; (iii) curcuminoid-enriched turmeric extract (CTE), typically obtained via precipitation at lower temperatures; ${ }^{4}$ (iv) curcuminoids-enriched materials (CEM), obtained from CTEs via chromatographic purification; (v) curcumin (1) as a single component, developed as highly purified, validated reference material and/or used for botanical standardization. As these five materials have distinct chemical compositions, with complexity decreasing from (i) to (v), their biological activity profiles have to be different. 
Existing confusion about the term "curcumin" and the CDS has already led to many incomparable and/or irreproducible reported biological results. ${ }^{2}$ While describing the phytochemical composition of any crude DS material is an established requirement for the accurate biological evaluation of the potential therapeutic value of DS. ${ }^{5}$ This basic tenet does not apply to the majority of CDS: their popularity as both a product and a research target, along with a frequent lack of specificity including in documentation, has blurred the lines between the single constituent, the plant extract stage, and all intermediate stages [see (i)-(v) above] of products. Pharmacokinetic properties complicate the matter further: curcumin (1; Scheme 1), the single compound, is very poorly absorbed, as are its congeners, demethoxycurcumin (2) and bisdemethoxycurcumin (3). Only traces of degradation compounds of $\mathbf{1}$ appear in the blood during the time when most of the curcumin is excreted in the feces. ${ }^{6,7}$ Owing to poor absorption of 1 into the bloodstream, piperine (4) has been recommended to enhance the curcumin absorption by 20 -fold ${ }^{8}$. However, this approach of metabolic inhibition is neither specific for $\mathbf{1}$, nor does it enhance $\mathbf{1}$ titers enough to reach pharmacologically relevant levels.

The Focus on Yellow Inspires Nefarious Adulteration Practices. Another major complicating factor in the CDS supply chain is the occurrence of adulteration. The addition of undeclared lower-cost ingredients to powdered turmeric root/rhizome and turmeric extracts is a major concern among regulators, healthcare professionals, and responsible members of the DS and herbal medicine industry. Reports of turmeric adulteration include substitution with other Curcuma species (e.g., C. zedoaria, C. xanthorrhiza, or C. aromatica), or the undeclared admixture of starches or yellow/orange dyes. Pigments such as Metanil Yellow, lead chromate, or Sudan Red have been added even to crude turmeric to produce a visually more appealing product. ${ }^{18}$ These practices appear to be more frequent in rural areas of India and Bangladesh. Particularly disturbing is a recent publication documenting elevated blood levels of lead in pregnant women living in rural Bangladesh due to the ingestion of turmeric adulterated with the lead chromate. ${ }^{19,20}$

The Consequences of Using a Single Compound to Represent a Whole Plant. In contemporary literature, the compound curcumin (1) is widely used as a sole "representative" of the plant, C. longa. This error has escalated to a point where products are sold in the marketplace that contains synthetic $\mathbf{1}$ rather than naturally derived $\mathbf{1}$, which are sometimes even labeled as $C$. longa extracts, allegedly standardized to a certain content of $\mathbf{1}$ and its congeneric curcuminoids $\mathbf{2}$ 
and 3. The discovery of this form of fraud has previously led to the investigation of its adverse effects in humans. ${ }^{9-11}$

Liver toxicity has been associated with products labeled as preparations that contain turmeric in a number of case reports. ${ }^{12-14}$ Moreover, between May and June of 2019, at least 21 cases of acute non-viral cholestatic hepatitis were reported in Italy and associated by authorities with over 20 different turmeric products. In a subset of products for which information was available, daily dosage recommendations ranged between 50 and $1200 \mathrm{mg}$ of "turmeric extract", labeled as being standardized to $95 \%$ curcuminoids, and containing 5 to $160 \mathrm{mg}$ of pepper (Piper nigrum, Piperaceae) extract, labeled as containing 95\% piperine. To prevent further cases, in July 2019, the Italian Ministry of Health eventually requested manufacturers of turmeric food supplements to include a warning about avoiding turmeric in patients with pre-existing liver conditions, in line with a recommendation by the European Medicines Agency (EMA) in $2018 .{ }^{15}$ Moreover, in cases of concomitant use of API-based medicines, ${ }^{16}$ the Italian Ministry of Health, through testing, had excluded contaminants, reaction by-products, solvent residues, and substitution with other plants as possible causes of the observed hepatotoxicities. However, the authorities also stated that there was no correlation between turmeric(!) itself and liver injury. ${ }^{17}$

Synthetic curcumin can be made for about one-third of the cost of natural curcumin, providing a financial incentive to unethical suppliers for diluting or replacing turmeric extracts or refined products with synthetic materials. A 2019 study using ${ }^{1} \mathrm{H}$ NMR and HPTLC to investigate the quality of 56 turmeric food supplements sold in Europe and the United States found that at least three of these supplements contained synthetic curcumin or synthetic curcuminoids based on the absence of the typically co-occurring turmeric compounds. ${ }^{21}$ In addition, a number of products claiming to contain piperine did not show the characteristic signals of the aromatic hydrogens in the ${ }^{1} \mathrm{H}$ NMR spectrum or the yellow band for the alkaloid in the HPTLC traces. ${ }^{22}$

In order to develop a more untargeted approach for recognizing "curcumin fraud", the present study considered known targeted analytical methods that have employed NMR, MS, and countercurrent separation methodologies. ${ }^{3,23-28}$ The only currently accepted proof that "curcumin" is synthetic utilizes isotope ratio mass spectrometry (IRMS). ${ }^{29,30}$ For the detection of synthetic $\mathbf{1}$, the determination of ${ }^{14} \mathrm{C}$ concentrations has proven useful as the synthesis of $\mathbf{1}$ uses petroleumbased starting materials, which are devoid of ${ }^{14} \mathrm{C} .{ }^{18}$ Contrarily, an equilibrium exists between ${ }^{14} \mathrm{C}$ intake via photosynthesis and its decay in living plants. Therefore, natural $\mathbf{1}$ and other natural 
turmeric compounds contain measurable amounts of ${ }^{14} \mathrm{C}$. The use of HPLC or HPTLC in the assessment of turmeric extract quality usually infers the presence of synthetic curcumin(oids) by the absence of the natural congeners or other compounds typical for turmeric extracts, e.g., arturmerone. The frequently observed admixture of other constituents such as (multi) vitamins and other plant extracts (e.g. those containing 4, or 4 itself) can produce HPLC peak overlap and, thereby, challenge even validated QC methods and provide additional rationale for the need to consider orthogonal analytical methods, such as NMR. Regardless of the analytical challenges associated with the untargeted detection of adulteration, economic calculations clearly show the (misguided) incentive of chemical synthesis over the purification of these compounds from a natural source.

\section{RESULTS AND DISCUSSION}

Extraction for CDS Adulteration Analysis. For non-polar extraction, a multi-solvent process was chosen (Figure S1, Supporting Information). Ten tablets were taken from each of the two commercial CDS batches A (7.98 g) and B (8.07 g). First, each batch was extracted twice,

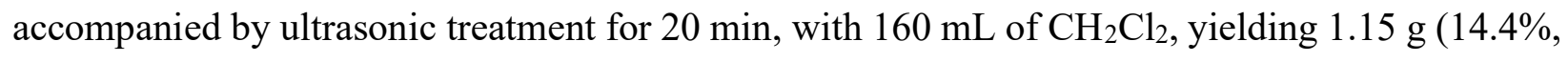
$\mathrm{w} / \mathrm{w})$ for batch A and $1.29 \mathrm{~g}(16.0 \%)$ for batch B. This was followed by MeOH extraction under the same conditions to yield $2.63 \mathrm{~g}(33.0 \%)$ and $3.22 \mathrm{~g}(39.9 \%)$, respectively (Figure S1, Supporting Information). The combined $\mathrm{CH}_{2} \mathrm{Cl}_{2}$ and $\mathrm{MeOH}$ extracts of each batch were chemically profiled by (U)HPLC-UV and qHNMR. The nonpolar extraction yield of $3.78 \mathrm{~g}$ (47.6\%) and $4.51 \mathrm{~g}(52.9 \%)$, respectively, showed that the two CDS batches from the same manufacturer had different constitutions, thereby revealing a potential quality (control) issue. For extraction of the declared polar constituents, namely vitamins, five tablets of each batch A (4.01 g) and B (4.05 g) were extracted twice, accompanied by ultrasonic treatment for 20 min, with 80 $\mathrm{mL}$ of $50 \% \mathrm{MeOH} / \mathrm{H}_{2} \mathrm{O}$, yielding $110 \mathrm{mg}$ (2.74\%) for batch A and 470mg (11.60\%) for batch B with $50 \% \mathrm{MeOH} / \mathrm{H}_{2} \mathrm{O}(20: 1, \mathrm{v} / \mathrm{w})$, again using the ultrasonic treatment for $20 \mathrm{~min}$ (Figure S2, Supporting Information). Qualitative and quantitative analysis of the extracts showed differences in composition for batches $\mathrm{A}$ and $\mathrm{B}$, as also reflected by the significant differences in yield, color, and chemical profiles (Figures S1-S3, Supporting Information).

Choice of Analytical Methodologies. Reflecting the abundance of HPLC instrumentation in laboratories worldwide, LC is employed widely for qualitative and quantitative (purity) analysis 
via relative referencing. The HPLC chemical profile for the non-polar extract (Figures S4 and S5, Supporting Information) is dominated by the curcumin (1) peak. The HPLC chromatogram also showed a small amount of piperine (4) and vitamin B2 (riboflavin; 6) as well as unidentified peaks of more polar compounds eluting around $6.5 \mathrm{~min}$ before 1 . Polar extracts were characterized by the presence of 1, 4, and 6. Vitamins B1 (thiamine; 5) and B6 (pyridoxine; 7) were too polar under the chosen conditions to be sufficiently retained for definitive identification (Figure S4, Supporting Information). The LC-based chemical profiles provided useful information relative to the available reference standards, but un-referenced peaks give little added information. Therefore, while LCbased targeted analysis can tentatively identify known chemical adulterants, its untargeted ability to recognize residual complexity is limited by the relatively high selectivity of the hyphenated detection method and/or the captured chromatographic polarity window.

Analysis by ${ }^{1} \mathrm{H}$ NMR can produce metabolomic fingerprints for chemometric evaluation and detection of adulteration of $C$. longa powders, ${ }^{21,31}$ and MS has been utilized to confirm the presence of compounds following structure elucidation by NMR. ${ }^{32}$ NMR offers a meaningful advantage due to its simple sample preparation, high reproducibility, and ability to detect diverse plant metabolites simultaneously. Counter-current separation (CCS) has been utilized for fractionation and isolation of $\mathbf{1}$ and its analogs from turmeric extracts. ${ }^{3,33-36}$ Notably, CCS can recover essentially $100 \%$ of loaded sample, ${ }^{37}$ because the stationary phase is liquid and, thus, all retained compounds can be readily recovered during an extrusion step or by washing. ${ }^{3}$ The present study employed a combination of CCS for sample preparation and quantitative ${ }^{1} \mathrm{H}$ NMR (qHNMR) to investigate the commercial CDS connected with the recently observed clinical hepatotoxicities.

NMR Identification of the Chemical Compositions. The CDS extracts were analyzed by ${ }^{1} \mathrm{H}$ NMR as an orthogonal method to (U)HPLC-UV (Figure S6, Supporting Information). The results from the non-polar extracts showed $\mathbf{1}$ as a major component, a trace amount of $\mathbf{4}$, and signals at $1.2 \mathrm{ppm}$ (aliphatic chain of fatty acid) indicated the presence of fatty material, likely adjuvants used as anti-caking agents (Figure 2 and Figure S6, Supporting Information). From the polar solvent extracts, only the presence of the $\mathbf{4 , 6}$, and polysaccharides (diagnostic signals in the range 2.8 - $5.8 \mathrm{ppm}$ ) with impurities was observed. However, as 5 (both labels claimed $2.7 \mathrm{mg} / 2$ tablets) and 7 (pyridoxine, both labels claimed $3.3 \mathrm{mg} / 2$ tablets) could not be detected in the CDS extracts (Figure S6, Supporting Information), either these two vitamins were difficult to extract from the 
tablets with the chosen solvents, or their contents in the tablets were below the LODs of the two analytical methods.

The results demonstrate that (U)HPLC-UV and ${ }^{1} \mathrm{H}$ NMR are complementary methods for creating chemical profiles. (U)HPLC-UV detected compounds with high UV absorption at $263 \mathrm{~nm}$ as chosen wavelength, but was blind to the presence of fatty and saccharide components. On the other hand, ${ }^{1} \mathrm{H}$ NMR not only observed the resonances belonging to the compounds detected via (U)HPLC-UV, but provided additional characteristic signals attributed to unknown components that were undetectable and/or unidentifiable by (U)HPLC-UV without the use of identical reference standards.

The labels of the investigated CDS declared that both products contained "95\% curcuminoids [plural!] from dried turmeric rhizome extracts $(500 \mathrm{mg} /$ tablet)", suggesting a natural source that contains multiple constituent curcuminoids. The labels also declared that 1 accounted for $76 \%$ of the content, while the remaining $19 \%$ of the $95 \%$ were due to 2 and $\mathbf{3}$. The ${ }^{1} \mathrm{H}$ NMR and (U)HPLCUV analyses of the non-polar extracts confirmed the presence of the major component, 1. However, when the ${ }^{1} \mathrm{H}$ NMR spectra of the non-polar extract are compared with the spectra of 2 and $\mathbf{3}$, it is evident that the extracts failed to show the characteristic signals of $\mathbf{2}$ and $\mathbf{3}$ in the 6.65 $-6.75 \mathrm{ppm}$ interval (Figure 1). Even when the baselines from the same spectra were enlarged in Figure 2, there was no evidence of signals that define 2 and 3 in the $6.65-6.75$ ppm interval. The NMR spectra indicated, therefore, that the source of the declared "curcuminoids" in the investigated commercial products lacked the residual complexity associated with naturally-derived 1. The dihydro derivatives indicative of a natural $\mathrm{CDS}^{3}$ were also absent. Inevitable residual complexity (go.uic.edu/residualcomplexity) results from the biosynthetic origin of the material and reflects the fact that a certain ("residual") amount of close analogs always accompany the major component as impurities, even after multiple large-scale enrichment or high-resolution laboratory purification steps.

Fingerprint Analysis of "Curcumin"-containing Dietary Supplements (CDS). Chemical fingerprints capture the complexity of a botanical DS, which is a result of their diverse chemical composition and as the diagnostically different abundance levels of their constituents. Chromatographic and spectroscopic fingerprints reveal known and/or unknown constituents by being targeted and/or untargeted, respectively. Of the most suitable untargeted techniques, NMR and FT-IR, ${ }^{39-41}$ NMR provides the more dispersed spectra with less signal overlap and, thereby, 
offers better capabilities for both targeted and untargeted analysis. This dual capability of (q)NMR, in combination with simultaneous quantification, was highlighted by the present outcomes of the present CDS analyses.

Mass Balance without Weighing. In order to further characterize the minor components of the residual complexity in the non-polar extracts, the 100\% qHNMR method was applied. This method is highly suitable for adulteration analysis as it forces the analyst to interpret as many signals as possible, ideally all observed signals (“100\%”). While this approach remains asymptotic by nature, it is still a powerful method that is generally underappreciated. The fact that the $100 \% \mathrm{qHNMR}$ method does not consider sample weight might appear to be counterintuitive for a quantitative method, but in fact, it bears a major advantage in quantitation asit is independent of weighing errors, including deviations from humidity effects. The main (systematic) error of the 100\% qHNMR method arises from chemical species, which the analysis cannot account for (properly) due to the lack of detectable ${ }^{1} \mathrm{H}$ nuclei and/or major disproportions in relative molecular weights. While the analyst must bear this in mind, it is a relatively minor limitation in the practice of NP and pharmaceutical analysis, at least in the authors' long-term experience. Notably, the unity response factor of qNMR remains a major intrinsic advantage for mass-balance and adulteration analysis, which outweighs the aforementioned systematic error.

Interestingly, 100\% methodology is ubiquitous in hyphenated LC-based quantitation. Thus, there is no reason why the well-established and easy-to-use 100\% "mass balance" quantitative evaluation method (i.e., integration of all visible peaks and determination of their ratio on a $100 \%$ basis) can be the gold standard in (U)HPLC and its many derivative methods, but could not be used equally widely in qHNMR. In fact, the 100\% qHNMR method should be considered more seriously as a very practical method that can be readily applied to almost any routine qualitative ${ }^{1} \mathrm{H}$ NMR data set. ${ }^{38}$

Practicability of $\mathbf{1 0 0 \%}$ qHNMR Fingerprint Analysis. NMR addresses the major limitation of HPLC quantitation with its need for calibration curves, which require the availability of identical reference standards for each target analyte. As an orthogonal method, qNMR methods offer both absolute (EC and IC qHNMR methods; abs-qNMR) and relative (100\% qHNMR method; relqNMR) quantification for purity and integrity analysis. The advantage of the applied 100\% qHNMR method is that it is both untargeted and independent of identical reference standards. Notably, it does not necessarily require knowledge of the particular mass of a detected molecular 
species as it measures the relative molarity. Moreover, it does so with a unity response factor, independently of knowing the mass and/or identity of the impurities. In the present study, data processing was performed using a previously optimized approach for qHNMR spectra, ${ }^{42}$ with the following parameters: a Lorentzian-Gaussian resolution enhancement (LG) with a Gaussian factor of $0.8 \mathrm{~Hz}$ and a line broadening factor of $-1.5 \mathrm{~Hz}$ was applied; all $64 \mathrm{k}$-sized time domain spectra were zero-filled to $512 \mathrm{k}$ data points to increase the digital resolution; a 5 th order polynomial fit was applied for the baseline correction.

Table 1 provides a summary of the findings from applying the 100\% qNMR method to the two non-polar extracts. The curcumin content was $95.16 \%$ and $92.57 \%$ in batches $A$ and $B$ respectively of the curcumin dietary supplement. In addition, batches A and B contained some $4(0.26 \%$ and $0.76 \%$ respectively) when signals that coincided with the signals produced by 4 reference material were integrated (Figure 4). The presence of curcumin(oid) degradation products in the non-polar extracts was confirmed by comparing their ${ }^{1} \mathrm{H}$ NMR spectra to those of purified natural curcumin (Figure 4). NMR spectra of natural 1 inevitably show the presence of degradation compounds as minor constituents and were utilized as reference for the same degradation products in the spectra of the investigated CDS. After the presence of $\mathbf{4}$ as well as likely degradation products was accounted for, unidentified aromatic impurities were observed and quantitated (Figure 4). In line with pharmacopoeial practices for unidentified impurities, the molecular weight of noncurcuminoid impurities was considered as the molecular weight of $\mathbf{1}$. Four different types of aromatic impurities were distinguished and their relative abundance measured via integration. Impurity 1 was associated with signals at $8.084,7.905$, and $7.870 \mathrm{ppm}$ that had identical integration values, impurity 2 was associated with the signal at $7.893 \mathrm{ppm}$, impurity 3 was associated with the signal at $7.642 \mathrm{ppm}$, and finally, impurity 4 was associated with signals at 7.205 and $7.003 \mathrm{ppm}$. It is very likely that there are other signals from the compounds that are obscured by overlapping absorbances. The origin of these signals was unknown. It is possible that these impurities may have been left behind by the starting materials and the reagents used in a synthetic procedure.

While the values measured for curcumin alone fell within the range of declaration (" $95 \%$ "), the findings were in conflict with regard to two other declared properties: (i) the specific content of 1 was much higher than declared, which is in line with the observation that $\mathbf{1}$ was the only curcuminoid present; and, (ii) the demethoxy ( $\mathbf{2}$ and $\mathbf{3}$ ) and dihydro curcuminoids, which constitute the inevitable residual complexity of $C$. longa products derived from nature, were below the 
detection limit of the qNMR method (signal to noise ratio $<1.5: 1$ ). This absence indicated that the material could not have been produced from plant material. Overall, the quantification results for 1, the absence of natural curcuminoid congeners, and the identification of unknown aromatic impurities implied that the investigated material was adulterated.

Countercurrent Fractionation of "Curcumin"-containing Dietary Supplements. In order to understand the chemical constituents more fully, chromatography was employed to distribute overlapping NMR signals into separate fractions. In a previous study, curcumin knock-out methodology was established using countercurrent separation (CCS) methodology. ${ }^{3}$ Specifically, centrifugal partition chromatography (CPC) was employed to fractionate the non-polar extract, using the two-phase solvent system composed of HDiMWat $\left(3: 7: 7: 3\right.$, hexanes- $\mathrm{CH}_{2} \mathrm{Cl}_{2}-\mathrm{MeOH}-$ water). The separation was performed in normal phase (ascending) mode, employing both elution and extrusion steps. The weight distribution of the resulting fractions (Figure 3) was as follows: Fr. 18.39 mg (7.1\%), Fr. 2 90.49 mg (76.0\%), Fr. 36.43 mg (5.4\%), Fr. 412.06 mg (10.1\%), Fr. $51.71 \mathrm{mg}(1.4 \%)$, Fr. $6<1 \mathrm{mg}(<1 \%)$. The curcumin knock-out materials obtained from both CDS were then compared with previously reported curcuminoid-enriched turmeric extracts. Using authentic reference compounds for comparison, Fr. 1 showed the presence of 4, Fr. 2 was identified as $\mathbf{1}$, and Frs. 3 and 4 represent minor amounts of $\mathbf{1}$ eluting at the tail-end of the chromatographic peak of 1. Observed NMR signal shifts are likely due to differences in residual water, concentration, and interaction/complexation with metal ions. However, the congeners $\mathbf{2}$ and $\mathbf{3}$, which are more highly retained in this SS, could not be detected at all, indicating the absence of a natural mixture of natural curcuminoids in the CDS. The polar fractions 5 and 6 showed the typical signals of saccharides (3.0 - $5.2 \mathrm{ppm}$ ) (Figure 3). CCS-based curcumin knock-out methodology is orthogonal to NMR, widens the dynamic range of subsequent analysis (see also Figure 3), and, by virtue of its liquid-liquid partition mechanism and 100\% recovery, can help confirm (or refute) the phytochemical integrity of a metabolome.

Breadth of Expected Chemical Variability. Plant metabolites exhibit wide structural variability, reflecting their wide range of functions for growth, survival, and reproduction. ${ }^{43-45}$ Plants also produce metabolites ahead of their death and to protect themselves from abiotic stress, harmful environmental pollution, and other external danger such as herbivores or for interspecies defense. ${ }^{46-48}$ Each plant produces specific chemical markers with minor phytochemical derivatives that are involved directly or indirectly in their survival. ${ }^{46,49,50}$ The production of all these 
constituents is affected by the condition of soil, cultivation, and time of harvest. ${ }^{51-53}$ While a single, ideally highly taxon-specific metabolite can be used as a reference standard for ID and DS fraud detection under specific circumstances, ${ }^{54,55}$ this approach does not capture the chemical variability of plants. This points to the contemptuous question, how natural chemical variation can be distinguished from adulteration.

As targeted analyses typically probe for the presence or abundance levels of "marker" metabolites, they are blind to adulteration outside of the probed very narrow (i.e., targeted) chemical window. In contrast, untargeted analysis has the capability of reaching outside this window. Distinguishing between acceptable and unacceptable differences can utilize detailed knowledge of natural variation (e.g., using a body of reference data from authentic materials, with visual or chemometric evaluation), but can also involve the recognition of more or less prominent analytical features that are either missing or extraneous or both. Examples of potentially missing features are the total absence of the signals for Curcuma oleoresins in CDS; examples of extraneous features are the presence of signals of aromatic compounds that are incompatible with the expected natural chemistry, such as those of synthetic precursors.

In addition to the consideration of targeted vs. untargeted analytical methods, regulatory aspects impact the potential answers to the above question. In the United States and the European Union, the rhizome of $C$. longa $\mathrm{L}$. and preparations derived from it are permitted products as set forth in the 1994 Dietary Supplements, Health, and Education Act (DSHEA 1994) and the Traditional Herbal Medicinal Products, a pan-EU harmonized legislation, respectively. Neither provision has specifications or requirements to prevent adulteration. In the EU, such preparations can also be used in food supplements, the regulation of which is largely the responsibility of individual member states. Countries such as Belgium, France, and Italy have developed lists of plants, preparations of which are permitted in food supplements. Such lists, however, do not address identification, authenticity, or prevention of adulteration. Specifically, before 2019, Italy had listed C. longa L. as a permitted plant, with no conditions of use. Since the 2019 adverse reports, mandatory warnings have been introduced, albeit with no further restrictions. ${ }^{56}$

However, no rules were introduced in reference to the potential adulteration of C. longa products. This applies to Belgium as well, which introduced further mandatory warnings in 2019, but also imposed a maximum limit of $500 \mathrm{mg}$ of curcuminoids per day for turmeric extracts with natural bioavailability, or equivalent amounts in case of enhanced bioavailability. ${ }^{57}$ The limit is 
based on food additive approval of "curcumin" (see the Introduction regarding general issues with the specificity of the nomenclature) as a food color and related uses. ${ }^{58}$ Other member states such as Ireland ${ }^{59}$ or France had or have also relied on maximum limits derived from food additive studies. Unfortunately, none of these regulatory actions took into account the authenticity of the plant source including the natural nature of the source. In terms of specific and new preparations, an application to market tetrahydro curcuminoids from turmeric has been recently submitted to the European Commission. ${ }^{60}$ With no apparent evidence of significant human consumption in the European Union before 1997, synthetic 1 is considered an unauthorized novel food, which cannot be marketed.

Methods for Targeted vs. Untargeted Detection of CDS Adulteration. Detecting the many different types of adulteration demands the use of orthogonal analytical methods. As the most commonly used, HPTLC and HPLC-UV/Vis can detect other Curcuma species and some of the colorants. ${ }^{24,61-65}$ The detection of lead chromate is challenging: it escapes routine testing methods due to its poor solubility in organic solvents and water, thus requiring targeted methods such as ICP-MS or flame atomic absorption spectroscopy. ${ }^{20,66}$ The Food Safety and Standards Authority of India also proposed an ashing/sulfuric acid-diphenylcarbazide test as a rapid screening for the lead but required confirmation by quantitative assays to confirm the level of contamination. ${ }^{67}$ Admixture of starches to powdered turmeric is readily detected microscopic powder examination using iodine stain or a xylene mount with full polarization. In the protocol here, starches are recognized as insoluble residues of extraction.

So far, the identification of undeclared synthetic curcumin has been achieved via MS experiments measuring the concentrations of ${ }^{14} \mathrm{C}$ isotopes. While a strict proof remains difficult, the present ${ }^{1} \mathrm{H}$ NMR method strongly inferred adulteration with synthetic curcumin and mislabeling of the product as turmeric extract based on the lack of residually complex, natural curcuminoid patterns, as well as the presence of several $\%$ of unidentified aromatic impurities that can be assigned to synthetic reagents and/or by products. To date, ${ }^{1} \mathrm{H}$ NMR has not been widely explored as a means for quality control of turmeric-based ingredients. ${ }^{68}$ However, its untargeted capabilities position NMR uniquely to solve many of the quality issues related to turmeric, its extracts and CDS in general. Conceptually, NMR can readily detect admixtures of and substitution with other Curcuma species, especially when the sample size is sufficient to allow multivariate statistics. Organic impurities, such as Metanil yellow and Sudan dyes, can be identified by their 
characteristic signals between 7.0 and $8.5 \mathrm{ppm}$ using ${ }^{1} \mathrm{H}$ NMR and were not detected in the investigated samples. ${ }^{69}$ As exemplified here, ${ }^{1} \mathrm{H}$ NMR can reveal a wealth of information about sample composition and, to some extent, the underlying manufacturing process by close examination (thus favoring the 100\% qNMR approach) of the residual complexity of the sample. ${ }^{3,69}$

More Comprehensive Coverage of Adulterants. The present work introduces the combined use of two methods with " $100 \%$ properties":

- the use of CCS permits quantitative separation with $100 \%$ sample recovery of the sample, due to its liquid-only nature; and

- the use of $100 \%$ qHNMR captures the entirety of hydrogen-containing chemical species in a (soluble) sample, due to its nuclear mechanism of detection.

In addition to being loss free, CCS is capable of partitioning the sample into a few yet chemically highly distinct fractions, which are amenable 100\% qHNMR, separately or after adequate recombination. Figure 3 shows the power of this off-line CCS-qHNMR combination in capturing both major and minor components by assessing the mass proportions of all sample components (via the masses of CCS fractions), providing information about the relative polarity of the constituents (via the CCS K-values), as well as giving details about their chemical constitutions (via the NMR $\delta$ scale and dispersion).

The study also shows that the combination of CCS and qNMR, two methods with analytically highly orthogonal characteristics, enables performance of identity and adulteration analysis that is both targeted and untargeted simultaneously. Accordingly, while representing a two-step process, such a combination widens the dynamic range of the analysis, thereby yielding unique insights that other single methodologies are unable to achieve. However, as shown above, qHNMR alone is still capable of making key conclusions about adulteration. As shown in Figure 4, even without CCS pre-fractionation, qHNMR detected the presence of aromatic impurities assigned to synthetic by-products, as well as the minor content of 4 . While the latter matched the declared constituent from $P$. nigrum, the CPC fractions enriched in $\mathbf{4}$ did not indicate the presence of additional alkaloids. Considering that 4 vastly dominates the alkaloid spectrum of natural P. nigrum extracts, distinction of natural extract from synthetic 4 is challenging at the low level of the declared ingredient in the investigated preparations. 
Putting DS Analysis and 100\% qHNMR Methodology in Context. Challenges in the quality control (QC) of botanical DS are associated with discerning adulterations, contaminants, and impurities as representing "chemical overlay's" of an already daunting variety of natural metabolites produced by the plants. Considering the chemical complexity of botanicals, the adulteration of DS enhances existing difficulties in producing reproducible biological results with them. The reductionist approach of using single or very few metabolites as biological placeholders and analytical reference standards exacerbates the overall challenge of detecting adulteration and fraudulent products sold in the marketplace.

This study shows how the universal and quantitative detection capabilities of (q)NMR, by itself or in combination with countercurrent pre-separation, provide an advanced foundation for concurrent targeted and untargeted DS adulteration assays. Demonstrated for two "curcumin"containing DSs that compromised the liver functions of several consumers in 2019, the approach is fit for recognizing adulteration of non-natural natural product ingredients (here: synthetic 1) in the presence of analytically confounding factors such as multi-vitamin and piperine fortification. These findings underscore the assertion that materials with limited supply, high demand, and/or high value drive economic motivations behind adulteration, ${ }^{28,70,71}$ which frequently are produced with additives (here: multi vitamins) that complicate the adulteration analysis rather than serving well-developed dietary or (implied) therapeutic benefits in the context of the main component. Considering the modest sample preparation requirements and ability to directly analyze extracts, the wealth of qualitative information achievable from 1D ${ }^{1} \mathrm{H}$ NMR spectra in tandem with their intrinsic quantitative capabilities via 100\% qHNMR evaluation make NMR a highly versatile adulteration assay that combines targeted and untargeted in a single method. The approach is ready for adoption to further applications in DS, food, herbal medicine, and drug analysis.

\section{EXPERIMENTAL SECTION}

General Experimental Procedures. Curcumin dietary supplements (Lot. A: 19B914, Lot. B: 18L823, Italy) claiming to be turmeric rhizome dry extract contained 95\% curcuminoid and 76\% curcumin, black pepper fruit dry extract contained 95\% piperine, vitamins; vitamin B1 (thiamine hydrochloride), B2 (riboflavin), B6 (pyridoxine hydrochloride), bulking agent; calcium carbonate, cellulose (pregelatinized corn starch) and anticaking agent; magnesium salts of fatty acids, silicon dioxide as ingredients. Reference compounds of natural curcumin (part\# 81025/lot 191793-106, 
Cayman Chemical Company, Ann Arbor, MI, USA), thiamine hydrochloride (part\# PHR10371G/lot LRAB2930, Sigma-Aldrich, St. Louis, MO, USA), riboflavin (part\# PHR1054-1G/lot LRAB3710, Sigma-Aldrich), pyridoxine hydrochloride (part\# PHR1036-500MG/lot LRAB3720, Sigma-Aldrich) were purchased for identification of chemical profile. Extraction solvents of CDS used were; $\mathrm{CH}_{2} \mathrm{Cl}_{2}$ (part\# 313000ACS/lot C17G20DRM-0000DCM, Greenfield Global, Shelbyville, KY, USA), MeOH (part\# 339000000/lot K18H15K10, Greenfield Global), water (Millipore Milli-Q gradient water purification system). UHPLC analyses were using a Shimadzu UFLC (Shimadzu Corp., Kyoto, Japna) Nexera UHPLC system equipped with a Diode Array Detector (SPD-M20-A), and fluorescence detector (RF-20A/20Axs), performed on a Kinetex 1.7 $\mu \mathrm{m}$ XB-C18 100Å column $(50 \times 2.1 \mathrm{~mm}$, Phenomenex, Torrance, CA, USA $)$. UHPLC-UV data analyses were processed with the Shimadzu Labsolution software package. HPLC grade solvents, acetonitrile (part\# 75-05-8/lot 183919) and water (part\# 7732-18-5/lot 163613) were purchased from Sigma-Aldrich with formic acid (part\# 64-18-6/lot B0538111B, Acros organic, NJ, USA). The sonicator, B-32H (Branson, CT, USA), was used for the extraction tablets of CDS. The ${ }^{1} \mathrm{H}$ NMR analyses (qHNMR) were performed on JEOL ECZ $400 \mathrm{MHz}$ equipped with a Super COOL probe (NM-Z161331TH5SC, JEOL Resonance Inc., Peabody, MA, USA). The NMR samples were diluted in $200 \mu \mathrm{L}$ of DMSO-d6 (part\# DML-10-10X1/lot 12G-464, Cambridge Isotope Laboratories, Inc., Tewksbury, MA, USA) transferred in 3 mm NMR tubes (part\# S-3-HT-7/lot D030915B, Norell, Morganton, NC, USA). The acquired data were processed using the Mnova NMR software package (v.14.1.0, MestreLab Research S.L., A Coruña, Spain).

Extract Preparation. For extraction of the non-polar constituents, ten tablets (\#A: $7.979 \mathrm{~g}$; \#B: $8.073 \mathrm{~g}$ ) of each CDS of batch A or B were extracted successively twice with $160 \mathrm{~mL}$ of $\mathrm{CH}_{2} \mathrm{Cl}_{2}$, subsequently extracted twice with $160 \mathrm{~mL}$ of $\mathrm{MeOH}$ by sonication. The $\mathrm{CH}_{2} \mathrm{Cl}_{2}$ extract combined with $\mathrm{MeOH}$ extract were combined to analyze the chemical profile by UHPLC-UV and ${ }^{1} \mathrm{H}$ NMR. For extracting the polar constituents, every five tablets (\#A: $4.009 \mathrm{~g}$; \#B: $4.051 \mathrm{~g}$ ) of curcumin dietary supplement for batch A or B were extracted successively with $80 \mathrm{~mL}$ of $50 \% \mathrm{MeOH} /$ water by sonication to analyze the chemical profile. The non-polar and polar extracts were analyzed for the chemical profile throughout UHPLC-UV and ${ }^{1} \mathrm{H}$ NMR. The non-polar extraction yielded 1.15 $\mathrm{g}(14.4 \%)$ of batch A and $1.29 \mathrm{~g}(16.0 \%)$ of batch B extracted by $\mathrm{CH}_{2} \mathrm{Cl}_{2}$ and obtained $2.63 \mathrm{~g}$ (33.0\%) for batch A and $3.22 \mathrm{~g}(39.9 \%)$ for batch B extracted by MeOH. The total extracts yield of non-polar extracts were $47.4 \%$ for batch $\mathrm{A}$ and $55.9 \%$ for batch $\mathrm{B}$. The polar extracts yielded 
$2.7 \%$ for batch $\mathrm{A}$ and $11.6 \%$ for batch $\mathrm{B}$ extracted by $50 \% \mathrm{MeOH} /$ water. For the quantitative NMR analyses, batch A (12.20 mg) and B (12.32 mg) as non-polar extracts and batch A (12.59 mg) and B (10.83 mg) as polar extracts, reference compounds; curcumin $(7.77 \mathrm{mg})$, piperine $(9.00 \mathrm{mg})$, thiamine hydrochloride $(7.59 \mathrm{mg})$, riboflavin $(2.33 \mathrm{mg})$ and pyridoxine $(7.10 \mathrm{mg})$ were each separately dissolved in $200 \mu \mathrm{L}$ of DMSO- $d_{6}$ then transferred in $3 \mathrm{~mm}$ NMR tube.

Acquisition of Chemical Profile of the Extracts. UHPLC-UV chemical profile analysis was performed with the Kinetex UHPLC column with solvents composed of (A) water and (B) MeCN both with $0.1 \%$ formic acid: from $5 \%$ B linearly increasing to $100 \%$ in $25 \mathrm{~min}$ at $0.7 \mathrm{~mL} / \mathrm{min}$. All extracts and reference compounds were prepared at $0.1 \mathrm{mg} / \mathrm{mL}$ with $\mathrm{MeOH} .2 \mu \mathrm{L}$ of the solution was injected for UHPLC-UV analyses under $190 \mathrm{~nm}$ of UV absorption.

The qHNMR data were acquired at $25^{\circ} \mathrm{C}$ under quantitative conditions using a $90^{\circ}$ single pulse experiment with a relaxation delay of $60 \mathrm{sec}$, a receiver gain of 46, and 64 scans. Post-acquisition data processing was performed using zero-filling of the $64 \mathrm{k}$ FID to $512 \mathrm{k}$ data points, a mild Lorentzian-Gaussian window function (Exponential factor -1.8, Gaussian factor 0.9 in GF mode), and baseline correction with a fifth-order polynomial function. The residual DMSO- $d_{5}$ signal was used for chemical shift referencing (2.5000 ppm). The NMR spectra were exported as a $\mathrm{jdx}$ (JCAMP-DX) file for quantitative processing in Mnova.

\section{SUPPORTING INFORMATION}

The Supporting Information is appended to this manuscript and contains the following elements: The non-polar (Figure S1) and polar (Figure S2) extraction processes of the "curcumin"-containing dietary supplements; the dried non-polar and polar solvent extracts (Figure S3); UHPLC-UV

chromatograms of the extracts (Figure S4); the ${ }^{1} \mathrm{H}$ NMR fingerprints (Figure S5); LC analysis of the impurities of the non-polar extracts (Figure S6).

\section{CORRESPONDING AUTHOR}

James B. McAlpine - Center for Natural Products Technologies (CENAPT), Program for Collaborative Research in the Pharmaceutical Sciences, Pharmacognosy Institute, and Department of Pharmaceutical Sciences, College of Pharmacy, University of Illinois at Chicago, Chicago, IL 60612, United States; orcid.org/0000-0003-1696-8828; Phone: +1 (312) 355-1949. E-mail: mcalpine@uic.edu. 
Guido F. Pauli - Center for Natural Products Technologies (CENAPT), Program for Collaborative Research in the Pharmaceutical Sciences, Pharmacognosy Institute, and Department of Pharmaceutical Sciences, College of Pharmacy, University of Illinois at Chicago, Chicago, IL 60612, United States; orcid.org/0000-0003-1022-4326

\section{OTHER AUTHORS}

Seon Beom Kim - Center for Natural Products Technologies (CENAPT), Program for Collaborative Research in the Pharmaceutical Sciences, Pharmacognosy Institute, and Department of Pharmaceutical Sciences, College of Pharmacy, University of Illinois at Chicago, Chicago, IL 60612, United States; orcid.org/0000-0001-8015-1404

Jonathan Bisson - Center for Natural Products Technologies (CENAPT), Program for Collaborative Research in the Pharmaceutical Sciences, Pharmacognosy Institute, and Department of Pharmaceutical Sciences, College of Pharmacy, University of Illinois at Chicago, Chicago, IL 60612, United States; orcid.org/0000-0003-1640-9989

J. Brent Friesen - Physical Sciences Department, Rosary College of Arts and Sciences, Dominican University, River Forest, IL, and Center for Natural Products Technologies (CENAPT), Program for Collaborative Research in the Pharmaceutical Sciences, Pharmacognosy Institute, and Department of Pharmaceutical Sciences, College of Pharmacy, University of Illinois at Chicago, Chicago, IL 60612, United States; orcid.org/0000-0003-1640$\underline{9989}$

Luca Bucchini - Hylobates, Consulting S.R.L., 00135 Rome, Italy

Stefan Gafner - American Botanical Council, Austin, TX 78723, United States

David C. Lankin - Center for Natural Products Technologies (CENAPT), Program for Collaborative Research in the Pharmaceutical Sciences, Pharmacognosy Institute, and Department of Pharmaceutical Sciences, College of Pharmacy, University of Illinois at Chicago, Chicago, IL 60612, United States; orcid.org/0000-0003-3166-5980

Shao-Nong Chen - Center for Natural Products Technologies (CENAPT), Program for Collaborative Research in the Pharmaceutical Sciences, Pharmacognosy Institute, and Department of Pharmaceutical Sciences, College of Pharmacy, University of Illinois at Chicago, Chicago, IL 60612, United States; orcid.org/0000-0003-0748-0863 
Guido F. Pauli - Center for Natural Products Technologies (CENAPT), Program for

Collaborative Research in the Pharmaceutical Sciences, Pharmacognosy Institute, and

Department of Pharmaceutical Sciences, College of Pharmacy, University of Illinois at Chicago,

Chicago, IL 60612, United States; orcid.org/0000-0003-1022-4326

\section{NOTES}

The authors declare no competing financial interests.

All raw data pertaining to the figures presented in this manuscript and its Supporting Information are made available at 10.7910/DVN/LOGPMI [ACTIVATION PENDING MANUSCRIPT ACCEPTANCE].

\section{ACKNOWLEDGEMENT}

The authors kindly acknowledge support by grant U41 AT008706 and supplement U41 AT008706-03S1 from NCCIH and ODS of the NIH.

\section{REFERENCES}

(1) Bisson, J.; McAlpine, J. B.; Friesen, J. B.; Chen, S.-N.; Graham, J.; Pauli, G. F. J. Med. Chem. 2016, 59, 1671-1690.

(2) Nelson, K. M.; Dahlin, J. L.; Bisson, J.; Graham, J.; Pauli, G. F.; Walters, M. A. J. Med. Chem. 2017, 60, 1620-1637.

(3) Friesen, J. B.; Liu, Y.; Chen, S.-N.; McAlpine, J. B.; Pauli, G. F. J. Nat. Prod. 2019, 82, 621-630.

(4) Antony, B. Treatment of Alzheimer's with a curcuminoid mixture and essential oil of turmeric having 45\% Ar-turmerone U.S. Patent 8,859,020 B2, 2014.

(5) Pauli, G. F.; Chen, S.-N.; Brent Friesen, J.; McAlpine, J. B.; Jaki, B. U. J. Nat. Prod. 2012, 75, 1243-1255.

(6) Ravindranath, V.; Chandrasekhara, N. Toxicology 1980, 16, 259-265.

(7) Ammon, H. P.; Wahl, M. A. Planta Med. 1991, 57, 1-7.

(8) Shoba, G.; Joy, D.; Joseph, T.; Majeed, M.; Rajendran, R.; Srinivas, P. S. Planta Med. 1998, 64, 353-356.

(9) Watson, E. EuroPharma to launch probe into synthetic vs natural curcumin. https://www.nutraingredients-usa.com/Article/2011/09/07/EuroPharma-to-launch-probeinto-synthetic-vs-natural-curcumin (accessed Sep 06, 2011).

(10) Daniells, S. Study highlights progress in turmeric supplement quality, but concerns around synthetic curcumin adulteration. https:/www.nutraingredients-

usa.com/Article/2018/06/18/Study-highlights-progress-in-turmeric-supplement-quality-butconcerns-around-synthetic-curcumin-adulteration (accessed Jun 18, 2018). 
(11) Sabinsa Corporation. Sabinsa takes action against Biotikon for synthetic curcumin adulteration. https:/www.sabinsa.com/press-release-2017/190-sabinsa-takes-action-againstbiotikon-for-synthetic-curcumin-adulteration (accessed Apr 11, 2017).

(12) Takikawa, H.; Murata, Y.; Horiike, N.; Fukui, H.; Onji, M. Hepatol. Res. 2009, 39, 427431.

(13) Lukefahr, A. L.; McEvoy, S.; Alfafara, C.; Funk, J. L. BMJ Case Rep. 2018, 2018.

(14) Luber, R. P.; Rentsch, C.; Lontos, S.; Pope, J. D.; Aung, A. K.; Schneider, H. G.; Kemp, W.; Roberts, S. K.; Majeed, A. Case Reports Hepatol. 2019, 2019, 6741213.

(15) Committee on Herbal Medicinal Products (HMPC). Assessment report on Curcuma longa L., rhizoma. https://www.ema.europa.eu/en/documents/herbal-report/final-assessmentreport-curcuma-longa-1-rhizoma-revision-1_en.pdf (accessed Sep 24, 2018).

(16) Bucchini, L. The 2019 Curcumin Crisis in Italy: What We Know so Far, and Early Lessons. 67th International Congress and Annual Meeting of the Society for Medicinal Plant and Natural Products Research (GA) in Cooperation with the French Society of Pharmacognosy AFERP. Innsbruck, Austra, Sep 1-5, 2019.

(17) Donelli, D.; Antonelli, M.; Firenzuoli, F. Intern. Emerg. Med. 2019, 15, 725-726.

(18) Bejar, E. Adulteration of turmeric (Curcuma longa) root and rhizome, and root and rhizome extracts. http://cms.herbalgram.org/BAP/BAB/TurmericRootandRhizomeandExtracts.html (accessed May, 2018).

(19) Forsyth, J. E.; Nurunnahar, S.; Islam, S. S.; Baker, M.; Yeasmin, D.; Saiful Islam, M.; Rahman, M.; Fendorf, S.; Ardoin, N. M.; Winch, P. J.; Luby, S. P. Environ. Res. 2019, 179, 108722.

(20) Forsyth, J. E.; Weaver, K. L.; Maher, K.; Islam, M. S.; Raqib, R.; Rahman, M.; Fendorf, S.; Luby, S. P. Environ. Sci. Technol. 2019, 53, 11429-11436.

(21) Chatzinasiou, L.; Booker, A.; MacLennan, E.; Mackonochie, M.; Heinrich, M. J. Herb. Med. 2019, 17-18, 100281.

(22) Booker, A.; Frommenwiler, D.; Johnston, D.; Umealajekwu, C.; Reich, E.; Heinrich, M. J. Ethnopharmacol. 2014, 152, 292-301.

(23) Gören, A. C.; Çıkrıkçı, S.; Çergel, M.; Bilsel, G. Food Chem. 2009, 113, 1239-1242.

(24) Jayaprakasha, G. K.; Rao, L. J. M.; Sakariah, K. K. J. Agric. Food Chem. 2002, 50, 36683672.

(25) Cao, Y.; Xu, R. X.; Liu, Z. J. Chromatogr. B Analyt. Technol. Biomed. Life Sci. 2014, 949950, 70-78.

(26) Priyadarsini, K. I. Molecules 2014, 19, 20091-20112.

(27) Dang, Y.-Y.; Li, X.-C.; Zhang, Q.-W.; Li, S.-P.; Wang, Y.-T. J. Sep. Sci. 2010, 33, 16581664.

(28) Song, W.; Qiao, X.; Liang, W.-F.; Ji, S.; Yang, L.; Wang, Y.; Xu, Y.-W.; Yang, Y.; Guo, D.-A.; Ye, M. J. Sep. Sci. 2015, 38, 3450-3453.

(29) Hong, E.; Lee, S. Y.; Jeong, J. Y.; Park, J. M.; Kim, B. H.; Kwon, K.; Chun, H. S. J. Sci. Food Agric. 2017, 97, 3877-3896.

(30) Drivelos, S. A.; Georgiou, C. A. Trends Analyt. Chem. 2012, 40, 38-51.

(31) Windarsih, A.; Rohman, A.; Swasono R. T. J. Appl. Pharm. Sci. 2018, 8, 75-81.

(32) Gilard, V.; Balayssac, S.; Tinaugus, A.; Martins, N.; Martino, R.; Malet-Martino, M. J. Pharm. Biomed. Anal. 2015, 102, 476-493.

(33) Friesen, J. B.; McAlpine, J. B.; Chen, S.-N.; Pauli, G. F. J. Nat. Prod. 2015, 78, 1765-1796.

(34) Inoue, K.; Nomura, C.; Ito, S.; Nagatsu, A.; Hino, T.; Oka, H. J. Agric. Food Chem. 2008, 
56, 9328-9336.

(35) Liu, Y.; Friesen, J. B.; Grzelak, E. M.; Fan, Q.; Tang, T.; Durić, K.; Jaki, B. U.; McAlpine, J. B.; Franzblau, S. G.; Chen, S.-N.; Pauli, G. F. J. Chromatogr. A 2017, 1504, 46-54.

(36) Liu, Y.; Friesen, J. B.; McAlpine, J. B.; Pauli, G. F. Planta Med. 2015, 81, 1582-1591.

(37) Malca Garcia, G. R.; Friesen, J. B.; Liu, Y.; Nikolić, D.; Lankin, D. C.; McAlpine, J. B.; Chen, S.-N.; Pauli, G. F. J. Chromatogr. A 2019, 1605, 360277.

(38) Pauli, G. F.; Chen, S.-N.; Simmler, C.; Lankin, D. C.; Gödecke, T.; Jaki, B. U.; Friesen, J. B.; McAlpine, J. B.; Napolitano, J. G. J. Med. Chem. 2014, 57, 9220-9231.

(39) Hall, R. D. New Phytologist. 2006, 169, 453-468.

(40) Simmler, C.; Kulakowski, D.; Lankin, D. C.; McAlpine, J. B.; Chen, S.-N.; Pauli, G. F. Adv. Nutr. 2016, 7, 179-189.

(41) Simmler, C.; Graham, J. G.; Chen, S.-N.; Pauli, G. F. Fitoterapia 2018, 129, 401-414.

(42) Pauli, G. F.; Jaki, B. U.; Lankin, D. C. J. Nat. Prod. 2005, 68, 133-149.

(43) Buchanan, B. B.; Gruissem, W.; Jones, R. L. Biochemistry and Molecular Biology of Plants; John Wiley \& Sons, 2015.

(44) Ibáñez, A. J.; Scharte, J.; Bones, P.; Pirkl, A.; Meldau, S.; Baldwin, I. T.; Hillenkamp, F.; Weis, E.; Dreisewerd, K. Plant Methods 2010, 6, 14.

(45) Dixon, R. A. Nature. 2001, 411, 843-847.

(46) Harborne, J. B. Ciba Found. Symp. 1990, 154, 126-134.

(47) Zaynab, M.; Fatima, M.; Sharif, Y.; Zafar, M. H.; Ali, H.; Khan, K. A. Microb. Pathog. 2019, 137, 103728 .

(48) Nejat, N.; Mantri, N. Curr. Issues Mol. Biol. 2017, 23, 1-16.

(49) Cowan, M. M. Clin. Microbiol. Rev. 1999, 12, 564-582.

(50) Waterman, P. G. Ciba Found. Symp. 2007, 171, 255-275.

(51) de Smet, P. A. G. M. Drug Inf. J. 1999, 33, 717-724.

(52) Sanzini, E.; Badea, M.; Dos Santos, A.; Restani, P.; Sievers, H. Food Funct. 2011, 12, 740746.

(53) Shenkar, J.; Griffiths, J. C. Clin. Pharmacol. Ther. 2018, 104, 423-425.

(54) Applequist, W. L.; Miller, J. S. Anal. Bioanal. Chem. 2013, 405, 4419-4428.

(55) Danezis, G. P.; Tsagkaris, A. S.; Camin, F.; Brusic, V.; Georgiou, C. A. Trends in Analyt. Chem. 2016, 85, 123-132.

(56) Integratori alimentari contenenti estratti e preparati di Curcuma longa. http://www.salute.gov.it/portale/news/p3_2_1_1_1.jsp?lingua $=$ italiano\&menu=notizie $\& p=d$ alministero\&id=3842 (accessed Jul 26, 2019).

(57) The Federal Public Service (FPS) Health, Commission d'avis des préparations de plantes. Avis Du 24 Octobre 2019 Rendu Par La Commission D'avis Des Préparations de Plantes Concernant L'utilisation de Compléments Alimentaires Contenant Des Curcuminoïdes; 2019.

(58) European Food Safety Authority, Scientific Opinion on the Re-evaluation of Curcumin (E 100) as a Food Additive. EFSA J. 2010, 8, 1679.

(59) Food Safety Authority of Ireland, Natures Aid Is Withdrawing All Batches of Its Turmeric due to High Levels of Curcumin, 2018.

(60) Sabinsa Europe GmbH. Tetrahydrocurcuminoids from Turmeric; European Commission, 2020.

(61) Dixit, S.; Khanna, S. K.; Das, M. ASimple 2-Directional High-Performance Thin-Layer Chromatographic Method for the Simultaneous Determination of Curcumin, Metanil 
Yellow, and Sudan Dyes in Turmeric, Chili, and Curry Powders. J. AOAC Int. 2008, 91, $1387-1396$.

(62) Windarsih, A.; Rohman, A.; Swasono, R. T. Int. J. Appl. Pharm. 2018, 10, 174.

(63) Mudge, E. M.; Brown, P. N. J. AOAC Int. 2018, 101, 203-207.

(64) Wichitnithad, W.; Jongaroonngamsang, N.; Pummangura, S.; Rojsitthisak, P. Phytochem. Anal. 2009, 20, 314-319.

(65) Paramapojn, S.; Gritsanapan, W. Acta Hortic. 2008, 786, 169-174.

(66) Cowell, W.; Ireland, T.; Vorhees, D.; Heiger-Bernays, W. Public Health Rep. 2017, 132, 289-293.

(67) Food Safety and Standards Authority of India (FSSAI). Manual of simple methods for testing of common adulterants in food. New Delhi, India. 2017, 1-55.

(68) Li, S. Pharm. Crop. 2011, 5, 28-54.

(69) Anibal, C. V. D.; Di Anibal, C. V.; Ruisánchez, I.; Pilar Callao, M. Food Chem. 2011, 124, $1139-1145$.

(70) Heffernan, C.; Ukrainczyk, M.; Gamidi, R. K.; Kieran Hodnett, B.; Rasmuson, Å. C. Org. Process Res. Dev. 2017, 21, 821-826.

(71) Skiba, M. B.; Luis, P. B.; Alfafara, C.; Billheimer, D.; Schneider, C.; Funk, J. L. Mol. Nutr. Food Res. 2018, 1800143. 


\section{SCHEMES, TABLES, AND FIGURES}<smiles>COc1cc(/C=C/C(=O)/C=C/c2ccc(O)c(OC)c2)ccc1O</smiles><smiles>COc1cc(/C=C/C(/C=C/c2ccc(O)cc2)=C/C(=O)O)ccc1O</smiles>
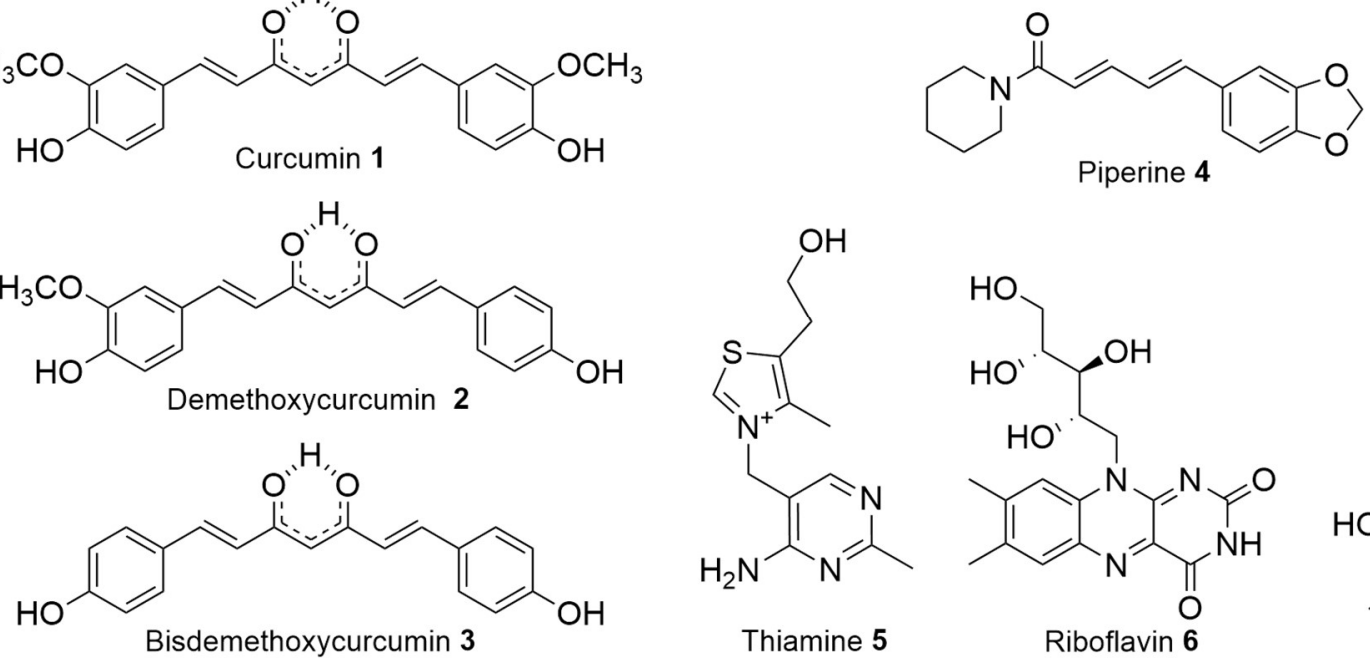

Scheme 1. the chemical structure of the ingredients of the curcumin dietary supplement studied. 


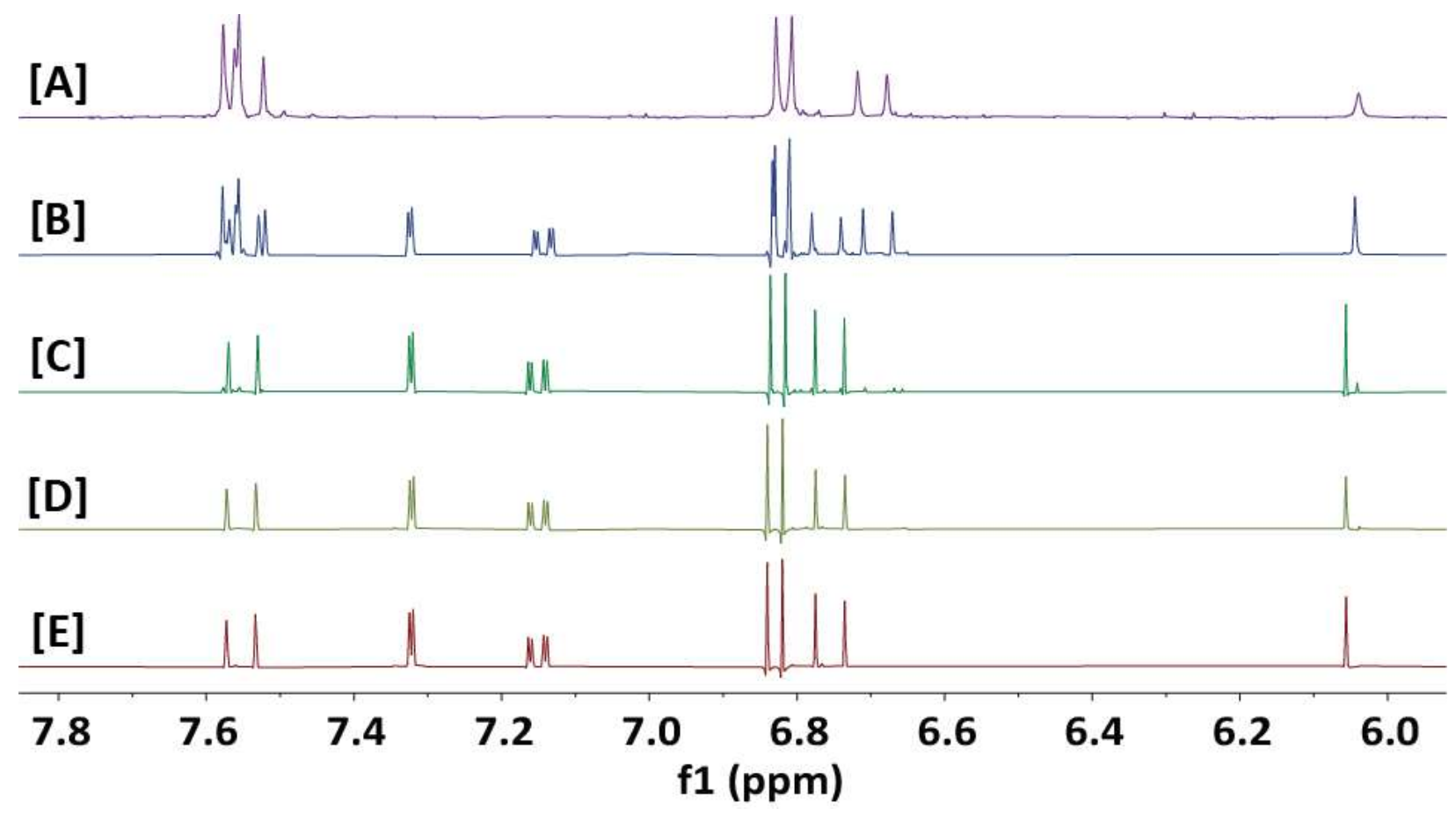

Figure 1. Comparison of the aromatic and olefinic range of the ${ }^{1} \mathbf{H}$ NMR profiles. The extracts of the "curcumin"-containing dietary supplement (CDS) (batches A [trace E] and B [trace D]) with those of authentic curcumin [1, trace C], demethoxycurcumin [2, trace B], bisdemethoxycurcumin $[3$, A] showed the lack of detectable natural congeners, thereby indicating adulteration with synthetic 1. 

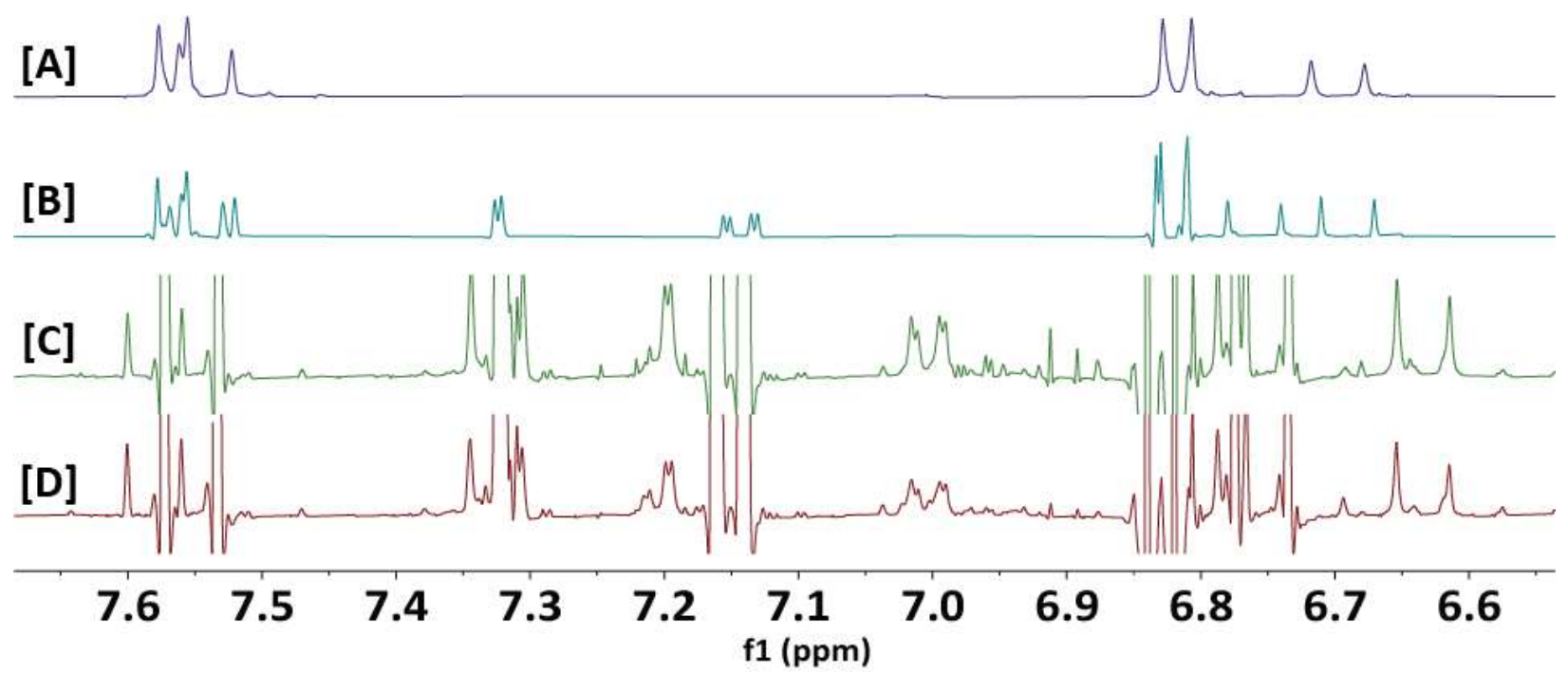

Figure 2. Residual complexity of non-natural "curcumin". The ${ }^{1} \mathrm{H}$ NMR spectra (processed with Lorentzian-Gaussian resolution enhancement) of the "curcumin"-containing dietary supplement extracts (batches A [trace D] and B [trace $\mathbf{C}$ ]) revealed the absence of the natural curcuminoid congeners, demethoxycurcumin [2, trace $\mathbf{B}]$ and bisdemethoxycurcumin $[\mathbf{3}$, trace $\mathbf{A}]$. Instead, several non-natural aromatic components were present that gave rise to AMX-type signal patterns similar to 1: $\sim 8 \mathrm{~Hz}$ doublets (impurity signal e.g. at $\delta_{\mathrm{H}} 8.58$ ) ; $\sim 2$ and $\sim 8 \mathrm{~Hz}$ doublets of doublets (e.g., impurity signal centered $\delta_{\mathrm{H}} 7.00$ ); $\sim 2 \mathrm{~Hz}$ doublets (e.g., impurity signal centered $\delta_{\mathrm{H}}$ 7.19); $\sim \mathrm{Hz}$ doublets (e.g., impurity signal centered at $\delta_{\mathrm{H}} 7.68$ ). The residual complexity patterns of the commercial samples were distinct from those of natural 1 or its natural precursors. 


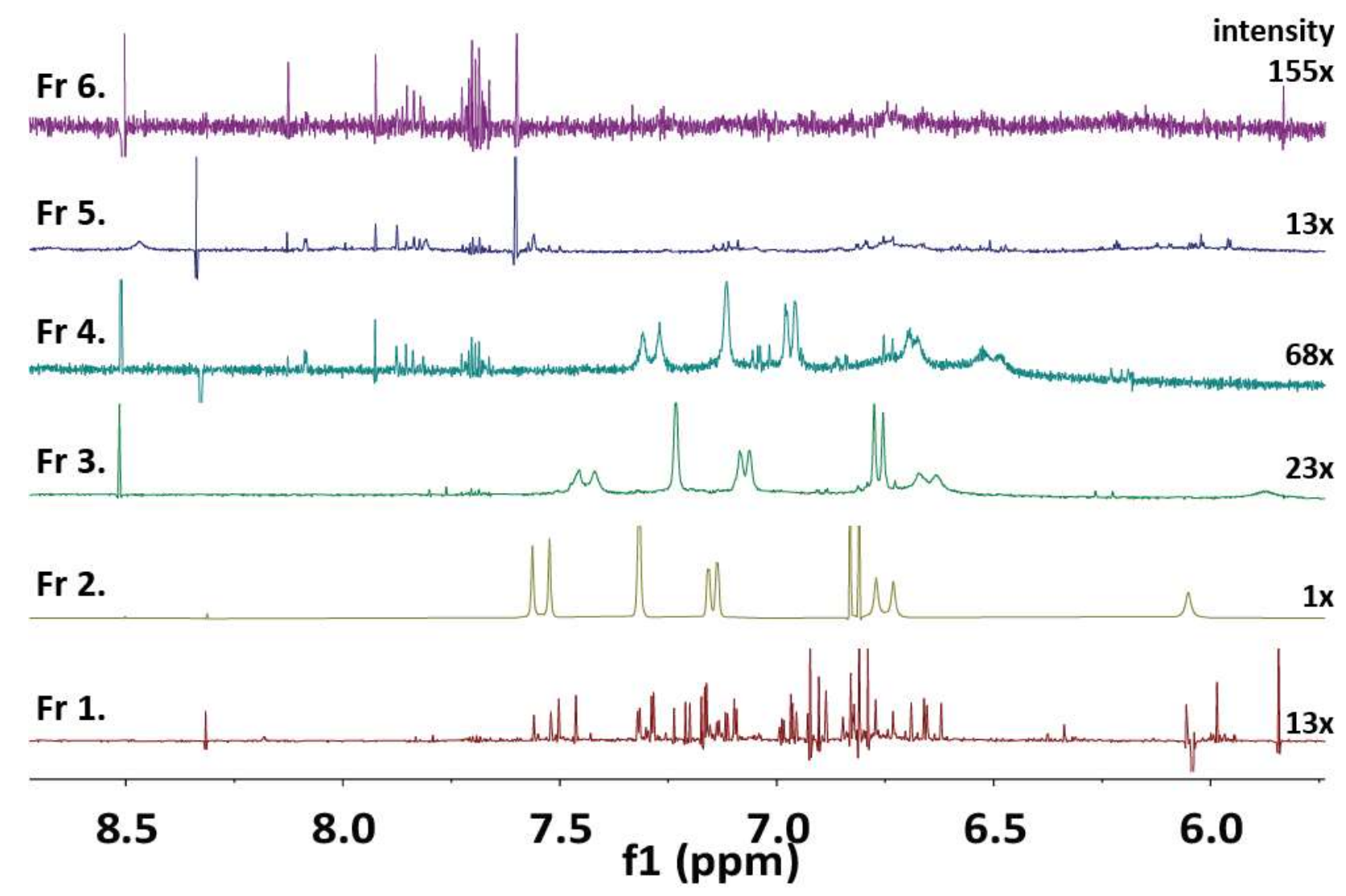

Figure 3. Aromatic range of the ${ }^{1} \mathrm{H}$ NMR spectra of the CPC fractions of one of the "curcumin"-containing dietary supplements. CPC was employed as an orthogonal and preseparation method for two reasons: first, to widen the chemical window and dynamic range for the targeted identification of the declared curcuminoids, vitamins, and piperine alkaloids; second, for the untargeted detection of unwanted constituents. The separation was performed as in normal phase (ascending) mode with hexanes- $\mathrm{CH}_{2} \mathrm{Cl}_{2}-\mathrm{MeOH}$-water (3:7:7:3) as a two-phase solvent system. The components eluted and eventually extruded (EECCC) in order of increasing polarity and were divided into seven fractions (see main text for weight distribution). The vertical scale of the spectra was chosen to show the major components. The intensity scaling factors reflect the vastly different abundance of the various components and highlight the achievable dynamic range enhancement of CCS pre-fractionation. 


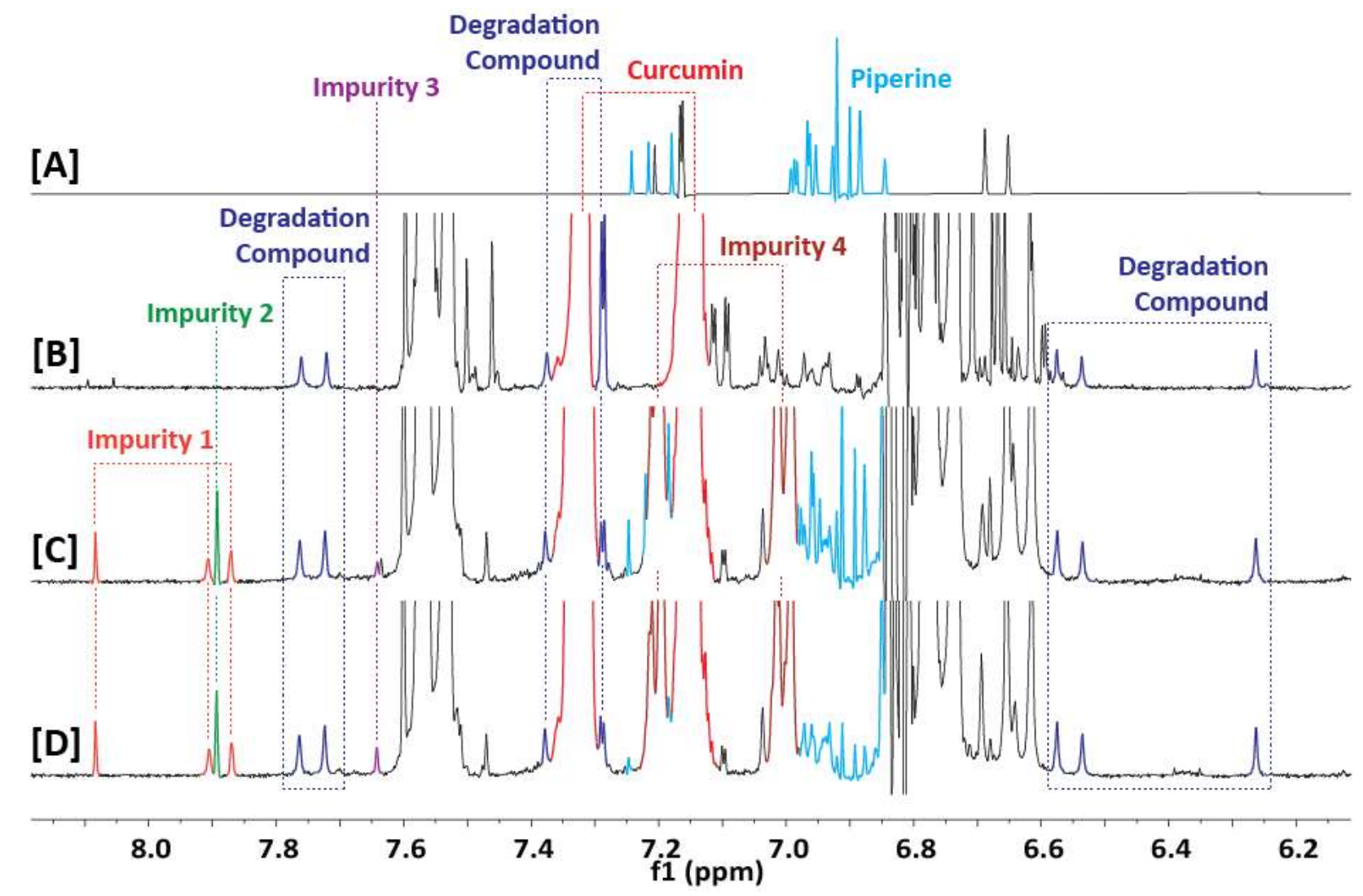

Figure 4. Assignment of the ${ }^{\mathbf{1}} \mathrm{H}$ NMR Aromatic Fingerprints in CDS. Both batches A [D] and $\mathrm{B}[\mathbf{C}]$ of the CDS contained the expected analytical targets: observation of the same major and minor signals as present in the spectrum of "pure" reference material (B) indicated the presence of an inevitable degradation compound in the main constituent, curcumin (1). The NMR fingerprints also contained the diagnostic signals of piperine (4; reference spectrum in A), despite its relatively low abundance at $0.54 \%$ and $2.01 \%$ for batch $\mathrm{A}$ and $\mathrm{B}$, respectively. In addition, four aromatic impurities were recognized in both CDS batches. Among the impurities compounds, impurity 4 revealed an AMX spin system which may synthetic precursor has the partial structure as the aromatic part of curcumin. For the relative qHNMR analysis, the integrals of the impurities were selected as indicated by the boxes (see Table 1). 
Table 1. The content of curcumin (1) in the commercial samples was determined using the $100 \%$ qHNMR approach, capturing both known and unknown constituents on a relative molarity basis and converting them to \% values by calculating the unknown as $\mathbf{1}$. The non-natural curcumin contained $95.16 \%$ and $92.57 \%$ in the curcumin dietary supplement, respectively, which contents of curcumin appear trying to make up with curcuminoid contents.

\begin{tabular}{|c|c|c|c|c|}
\hline \multirow[b]{2}{*}{ component } & \multicolumn{4}{|c|}{ quantities of curcumin in the dietary supplements } \\
\hline & $\delta_{\mathrm{H}}$ & batch A (ppm, \% & & average integral value \\
\hline curcumin & $7.323(97.78)$ & $7.151(100.00)$ & & 98.89 \\
\hline impurity 1 & $8.084(0.13)$ & $7.905(0.13)$ & $7.870(0.14)$ & 0.13 \\
\hline impurity 2 & $7.893(0.25)$ & & & 0.25 \\
\hline impurity 3 & $7.642(0.10)$ & & & 0.10 \\
\hline impurity 4 & $7.205(4.51)$ & $7.003(4.58)$ & & 4.55 \\
\hline \multicolumn{5}{|c|}{ curcumin content $(\%)$ : 95.2} \\
\hline & \multicolumn{3}{|c|}{ batch B (ppm, \%) } & average integral value \\
\hline curcumin & $7.323(97.40)$ & $7.151(100.00)$ & & 98.70 \\
\hline impurity 1 & $8.084(0.11)$ & $7.905(0.11)$ & $7.870(0.12)$ & 0.11 \\
\hline impurity 2 & $7.893(0.24)$ & & & 0.24 \\
\hline impurity 3 & $7.642(0.08)$ & & & 0.08 \\
\hline impurity 4 & $7.205(7.49)$ & $7.003(7.48)$ & & 7.49 \\
\hline curcumin $\mathrm{c}$ & tent (\%): 92.6 & & & \\
\hline
\end{tabular}




\section{The Untargeted Capability of NMR Recognizes Adulterated Natural Products}

Seon Beom Kim, Jonathan Bisson, J. Brent Friesen, Luca Bucchini, Stefan Gafner, David C. Lankin, Shao-Nong Chen, Guido F. Pauli, James B. McAlpine*

- SUPPORTING INFORMATION 


\section{TABLE OF CONTENTS}

Figure S1. Non-polar extraction workflow of the investigated two batches of curcumin dietary supplements (CDSs).

Figure S2. Polar extraction workflow of the investigated two batches of curcumin dietary supplements (CDSs).

Figure S3. The dried non-polar and polar solvent extracts from the investigated curcumin dietary supplements (CDSs).

Figure S4. UHPLC-UV chemical profiling of the investigated curcumin dietary supplements (CDSs) and five authentic reference compounds.

Figure S5. The ${ }^{1} \mathrm{H}$ NMR chemical fingerprints of the investigated curcumin dietary supplements (CDSs).

Figure S6. LC analysis of impurities in the non-polar extracts of the investigated curcumin dietary supplements (CDSs). 
Figure S1. Non-polar extraction workflow of the investigated two batches of curcumin dietary supplements (CDSs).

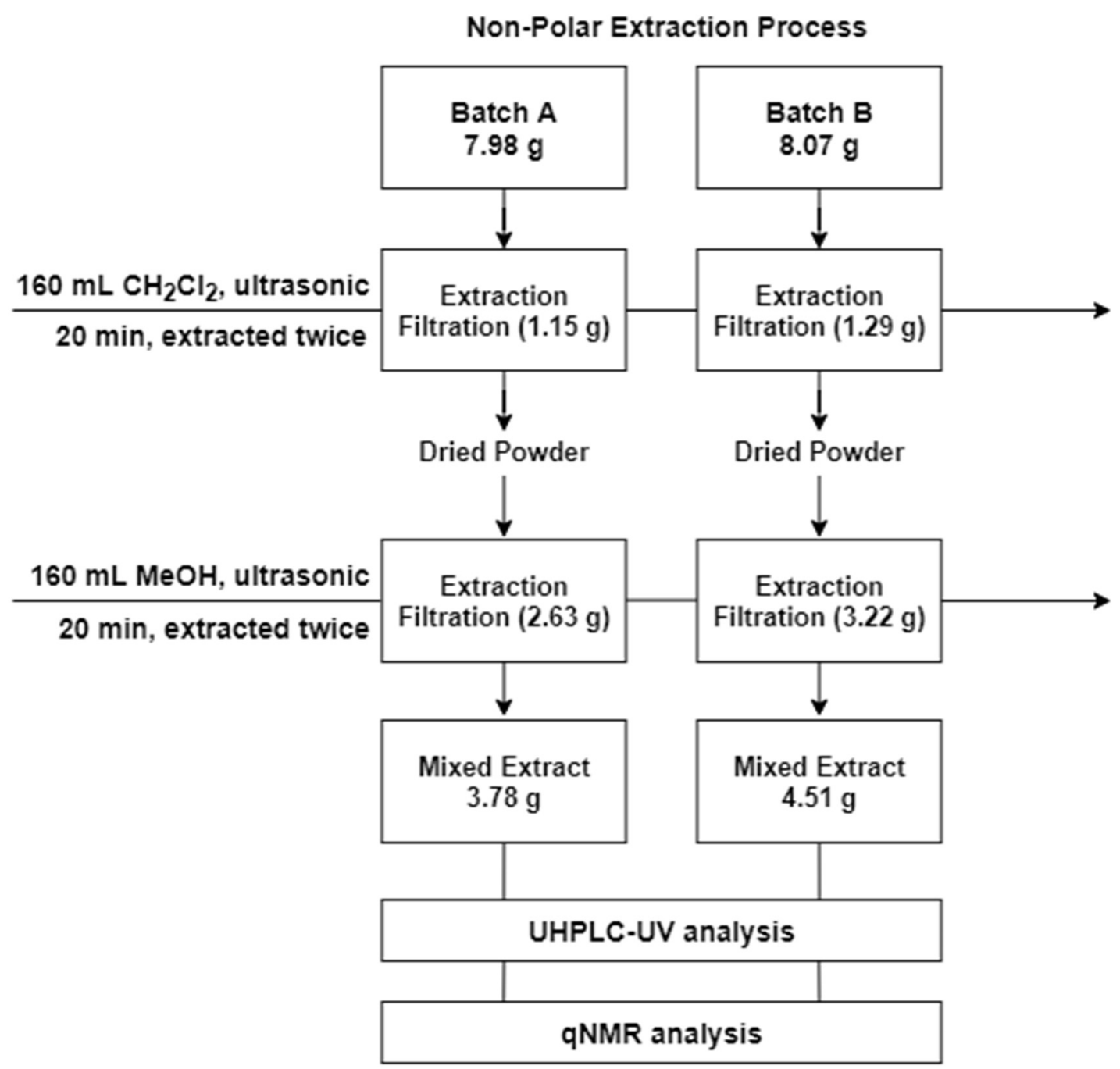

Ten tablets of batch A and B were prepared for the non-polar extraction process. Extracted twice with sonication and maceration using two different solvent systems, $\mathrm{CH} 2 \mathrm{Cl} 2(20: 1$, v/w) and $\mathrm{MeOH}(20: 1, \mathrm{v} / \mathrm{w})$. The combined extracts were utilized for the identification of chemical profile and phytochemical fingerprint using (U)HPLC-UV and qNMR analysis techniques. 
Figure S2. Polar extraction workflow of the investigated two batches of curcumin dietary supplements (CDSs).

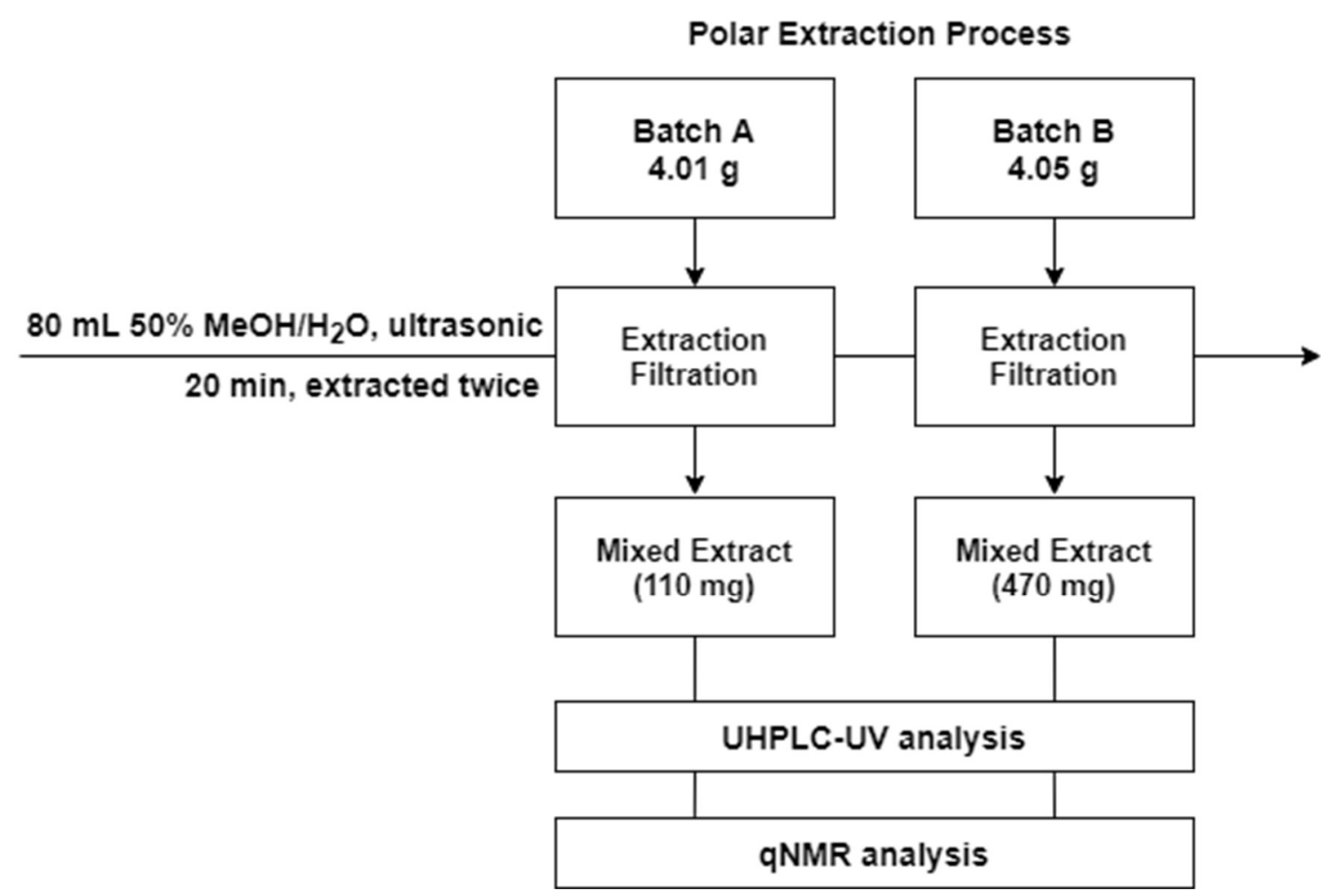

Five tablets of batch A and B were prepared for the polar extraction process. Extracted twice with sonication and maceration using $50 \% \mathrm{MeOH} / \mathrm{H}_{2} \mathrm{O}(20: 1, \mathrm{v} / \mathrm{w})$. The extracts were utilized for the identification of chemical profile and phytochemical fingerprint using (U)HPLC-UV and qNMR analysis techniques. 
Figure S3. The dried non-polar and polar solvent extracts from the investigated curcumin dietary supplements (CDSs).

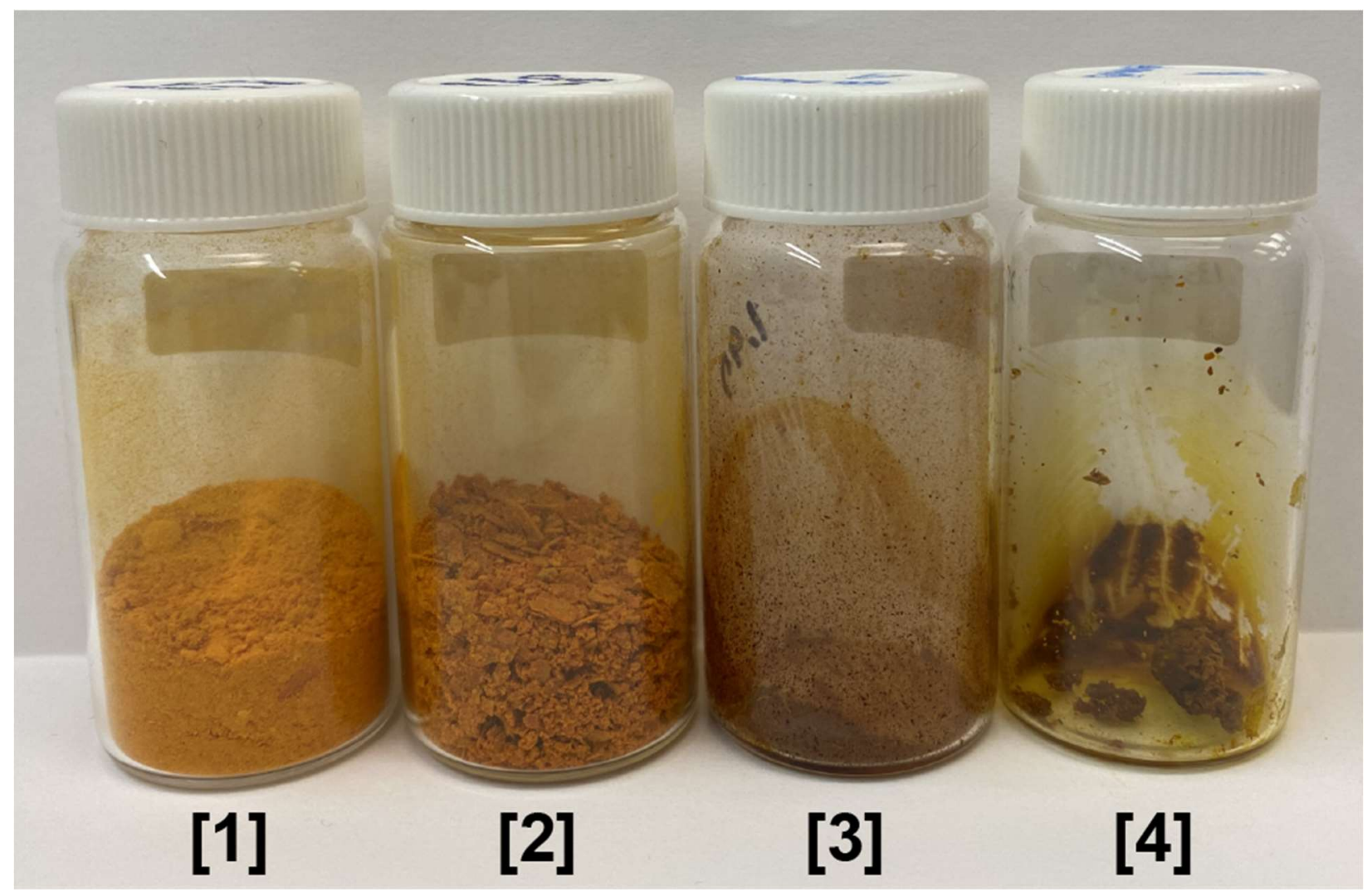

Extracts of CDS samples batch A and B show significant color and yield differences. The nonpolar solvent extract obtained weighed $3.78 \mathrm{~g}(47.6 \%)$ for batch A [1] and $4.51 \mathrm{~g}(52.9 \%)$ for batch B [2]. The polar solvent extracts confirmed batch disparity: $110 \mathrm{mg}(2.74 \%)$ were obtained for batch A [3] and $470 \mathrm{mg}(11.60 \%)$ for batch B [4]. 
Figure S4. UHPLC-UV chemical profiling of the investigated curcumin dietary supplements (CDSs) and five authentic reference compounds.
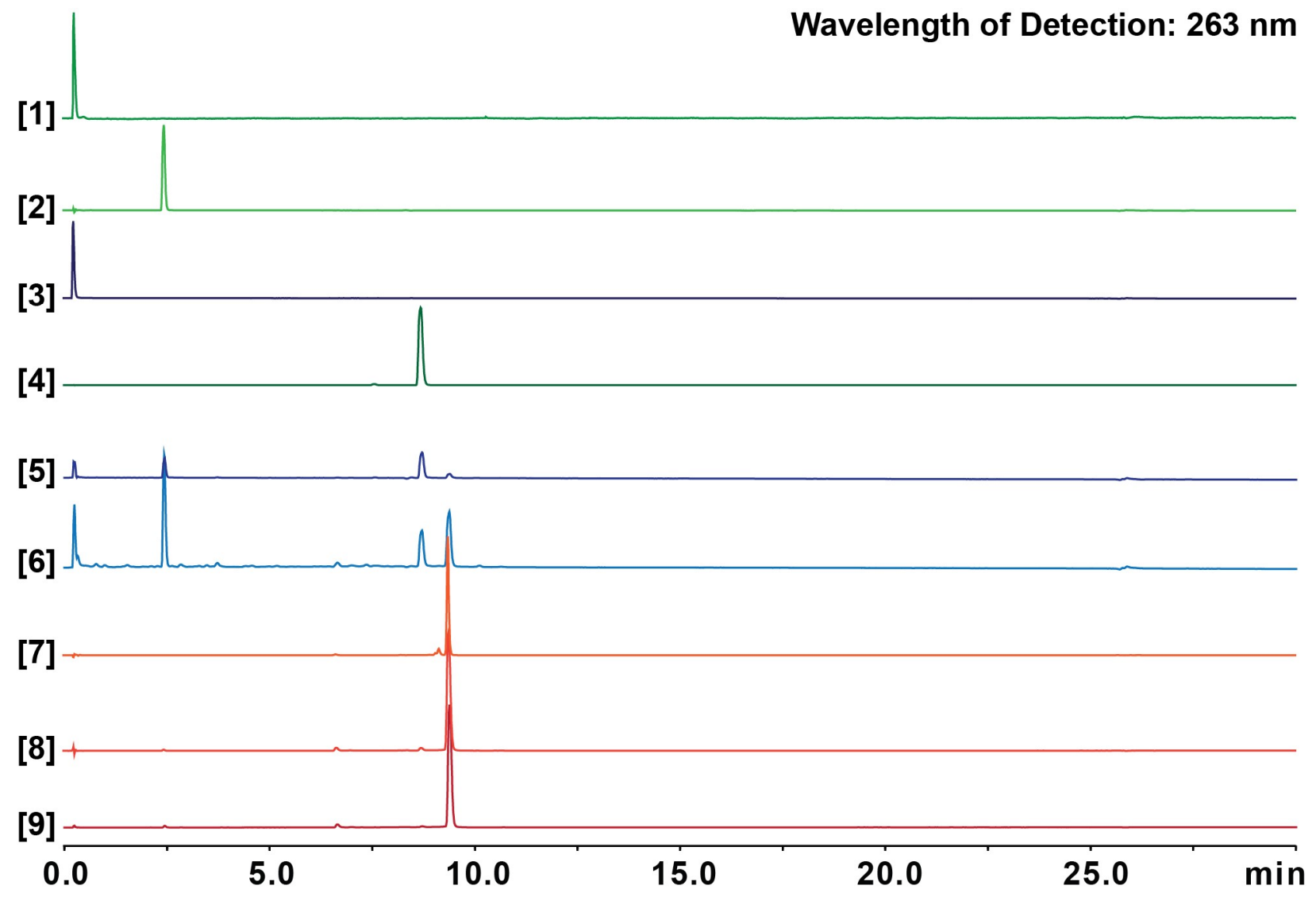

Stacked UHPLC-UV chromatograms (detected: $263 \mathrm{~nm}$ ) of curcumin dietary supplement extracts: batches $\mathrm{A}$ [9 and 6] and $\mathrm{B}$ [8 and 5] extracted with $100 \% \mathrm{CH}_{2} \mathrm{Cl}_{2}$ and $100 \% \mathrm{MeOHand}[50 \%$ $\mathrm{MeOH} / \mathrm{H}_{2} \mathrm{O}$ ), respectively. Reference compounds were: curcumin [7], piperine [4], vitamins B1 (thiamine) [3], B2 (riboflavin) [2] and B6 (pyridoxine) [1]. 
Figure S5. LC analysis of impurities in the non-polar extracts of the investigated curcumin dietary supplements (CDSs).

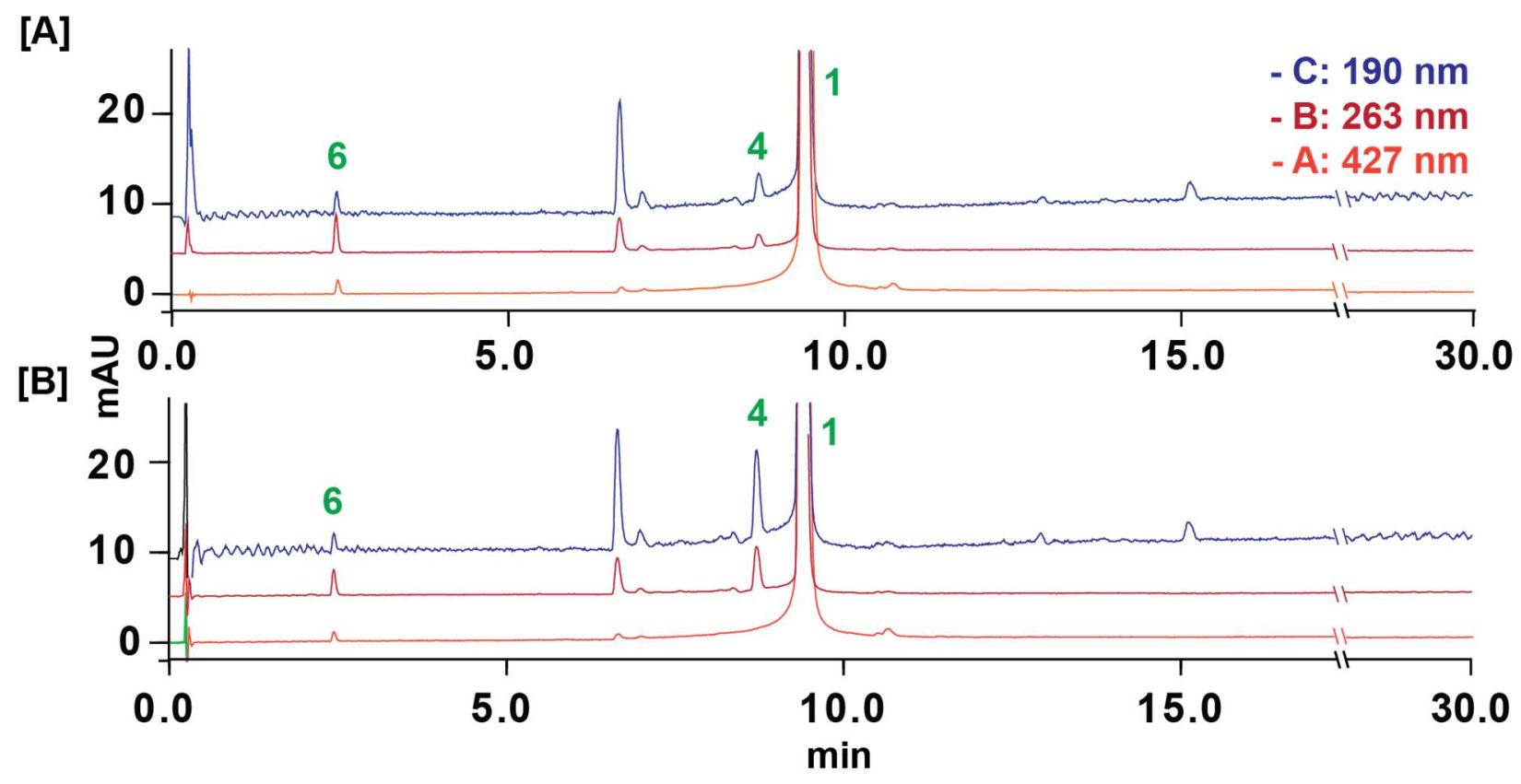

(U)HPLC-UV analyzed non-polar extracts for batch A $[\mathbf{A}]$ and B $[\mathbf{B}]$ on the three different UV absorptions. curcumin (1), piperine (4) and riboflavin (6) were identified by retention time and UV absorptions. The UV spectrum showed that the absence of curcuminoids constituents in the CDS extracts, together with unidentified residual peaks were observed. 
Figure S6. The ${ }^{1} \mathrm{H}$ NMR chemical fingerprints of the investigated curcumin dietary supplements (CDSs).

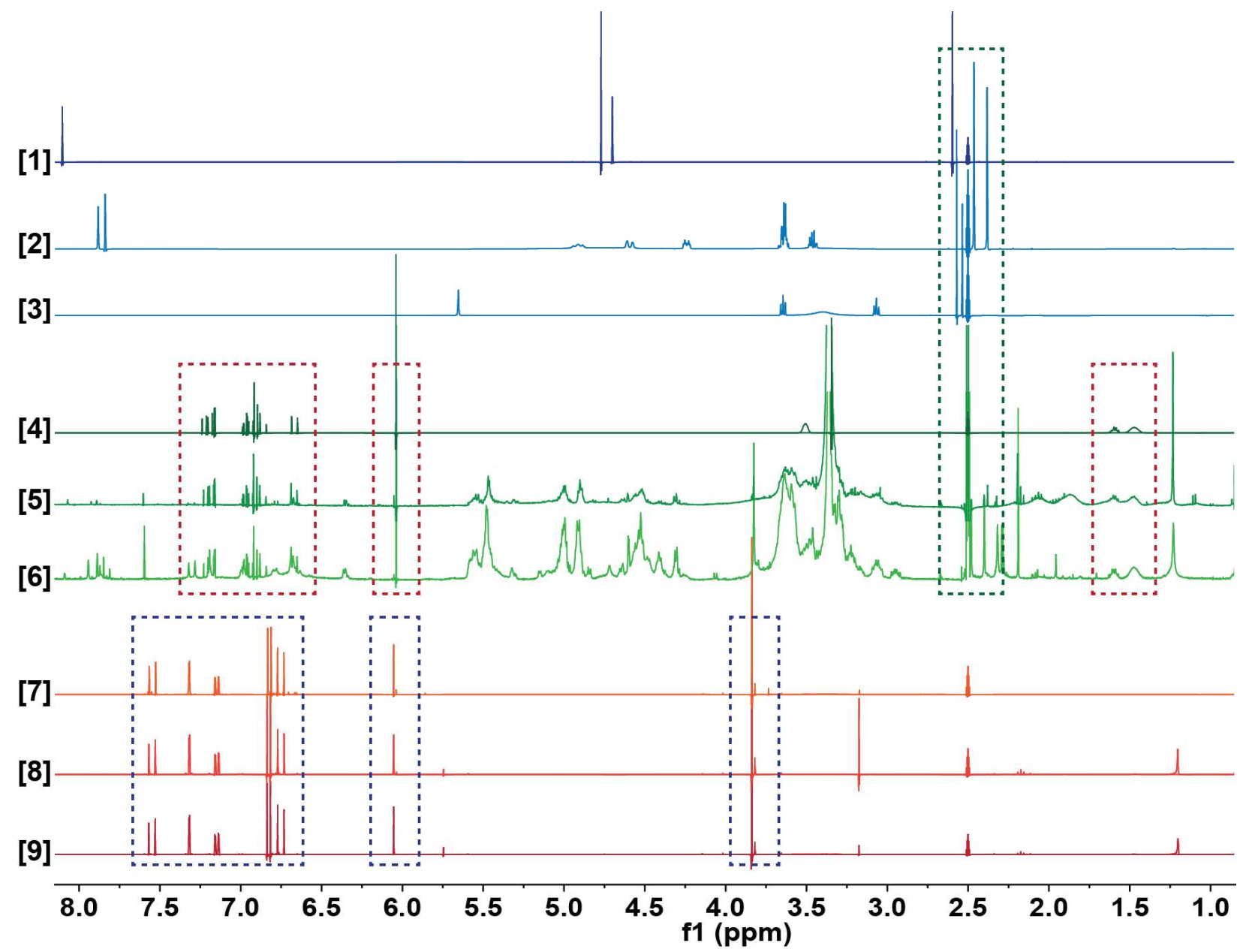

Stacked ${ }^{1} \mathrm{H}$ NMR (DMSO- $d_{6}, 400 \mathrm{MHz}$ ) spectra of curcumin dietary supplement extracts: batches $\mathrm{A}$ [9 and 6] and $\mathrm{B}$ [8 and 5] extracted with $100 \% \mathrm{CH}_{2} \mathrm{Cl}_{2}$ and $100 \% \mathrm{MeOH}$ and $\left[50 \% \mathrm{MeOH} / \mathrm{H}_{2} \mathrm{O}\right.$ ), respectively. Reference compounds were: curcumin in trace [7], piperine in trace [4], vitamins B1 (thiamine) in trace [3], B2 (riboflavin) in trace [2], and B6 (pyridoxine) in trace [1]. 